\title{
TRAPPED MODES IN AN ELASTIC PLATE WITH A HOLE
}

\author{
C. FÖRSTER AND T. WEIDL
}

In the memory of our dear teacher M. Sh. Birman

\begin{abstract}
For an infinite linear elastic plate with stress-free boundary, the trapped modes arising around holes in the plate are investigated. These are $L^{2}$-eigenvalues of the elastostatic operator in the punched plate subject to Neumann type stress-free boundary conditions at the surface of the hole. It is proved that the perturbation gives rise to infinitely many eigenvalues embedded into the essential spectrum. The eigenvalues accumulate to a positive threshold. An estimate of the accumulation rate is given.
\end{abstract}

\section{§1. INTRODUCTION}

1.1. We consider a homogeneous and isotropic linear elastic medium in the domain $G=\mathbb{R}^{2} \times J$ with $J=\left(-\frac{\pi}{2}, \frac{\pi}{2}\right)$. For functions $u, v \in H^{1}\left(G ; \mathbb{C}^{3}\right)$, we set

$$
\epsilon(u)=\frac{1}{2}\left(\nabla u+(\nabla u)^{T}\right) \quad \text { and } \quad\langle\epsilon(u), \epsilon(v)\rangle_{\mathbb{C}^{3 \times 3}}=\sum_{i, j=1}^{3} \epsilon_{i j}(u) \overline{\epsilon_{j i}(v)} .
$$

Then the quadratic form

$$
a[u]=2 \int_{G}\langle\epsilon(u), \epsilon(u)\rangle_{\mathbb{C}^{3 \times 3}} \mathrm{~d} x
$$

induces the elastostatic operator $A$ for materials with zero Poisson ratio; $A$ corresponds to the differential expression

$$
-(\Delta+\operatorname{grad} \operatorname{div})
$$

with stress-free (Neumann-type) boundary conditions 1 The operator $A$ has purely absolutely continuous spectrum filling the nonnegative half-line.

Now, consider a punched plate $\Omega^{c}=G \backslash \bar{\Omega}$ with a hole $\Omega=\Omega_{0} \times J$, where $\Omega_{0} \subset \mathbb{R}^{2}$ is a bounded Lipschitz domain. Let $A_{\Omega^{c}}$ be the elastostatic operator corresponding to the differential expression (1.2) on the outer domain $\Omega^{c}$ subject to stress-free (Neumanntype) boundary conditions. This geometric perturbation does not change the location of the essential spectrum, but it gives rise to a somewhat unexpected trapping effect. As the main result of this paper we prove the existence of infinitely many eigenvalues $\left\{\nu_{k}\right\}_{k \in \mathbb{N}}$ of $A_{\Omega^{c}}$, embedded into the essential spectrum, which accumulate at a certain threshold $\Lambda>0$, and we compute the accumulation rate of these trapped modes. We establish the formula

$$
\ln \left(\Lambda-\nu_{k}\right)=-2 k \ln k+o(k \ln k) \quad \text { as } \quad k \rightarrow \infty .
$$

2010 Mathematics Subject Classification. Primary 74B05.

Key words and phrases. Elasticity operator, trapped modes.

${ }^{1}$ Here we have chosen a suitable set of units such that Young's modulus $E$ fulfills $E=2$. Moreover, we point out that the special choice of zero Poisson ratio is essential for the results of this paper. 
This paper is related to an extensive series of works on trapped modes in perturbed quantum [1]-[5], acoustic [6]-[10], and elastic waveguides [11]-[15].

The existence of eigenvalues near a lower threshold of a suitable spectral branch of the operator can be shown by reducing the problem to the corresponding scattering channel via separation of variables. The spectral structure of the model near the threshold is then given by the behavior of an eigenvalue branch of a parameter dependent SturmLiouville problem. In the acoustic and the quantum case, this usually turns out to be a nondegenerate quadratic minimum. Therefore, one can carry out a weak-coupling analysis in the spirit of [16] for an effective Laplacian.

However, elastic waveguides are given by systems of partial differential equations. The structure of the spectral minimum of a scattering channel is described by the behavior of an eigenvalue branch of a certain effective matrix-valued Sturm-Liouville problem. It turns out that for the scattering channel of interest this minimum is strongly degenerate, what leads to a weak-coupling analysis of a pseudodifferential operator with degenerate symbol in the spirit of [17]. In particular, the minimum will be attained on the circle $\left\{\xi \in \mathbb{R}^{2}:|\xi|=\varkappa\right\}$ for some $\varkappa>0$. This means that there are infinitely many wave functions in the spectral minimum, which may turn into eigenfunctions in the case of perturbation. A corresponding analysis for additive perturbations was carried out in [15]. Here we consider the case of geometric perturbations by Neumann-type boundary conditions.

First, we transform the problem with boundary perturbation into a problem with additive perturbation. For this, we pass to the resolvents of the elasticity operators. Then the additive perturbation is described by suitable operators of elastic potential theory. The main task is now to analyze the spectral behavior of this perturbation operator.

As in [15, we need to deal with super-polynomial accumulation rates of the eigenvalues. Note that such accumulation rates have recently been obtained also in other physical settings; see, e.g., 18]-20. In general, they rest on the combination of compactly supported perturbations applied to strongly degenerate operators.

The paper is structured as follows. In $\sqrt{2}$ we state the problem in detail. In $\S 3$ we describe the spatial and internal symmetries of the problem that allow us to distinguish embedded eigenvalues from the surrounding essential spectrum. Considering the elasticity problem for a suitable symmetry case, we give a short description of its degenerate spectral minimum. See [14, 15, for a detailed investigation.

In $\$ 4$ we state our main results. There we also give a proof of the existence result for holes, which is based on the results of [15]. In \$5] we provide the formulation of the perturbation problem in terms of the inverse operators. For the investigation of the accumulation rate we need further auxiliary material, which is presented in $\sqrt[6]{6}$. There we introduce general classes of operators that allow us to handle super-polynomial eigenvalue asymptotics beyond the well-known $\Sigma_{p}$-classes for compact operators. Furthermore, we state a special type of Birman-Schwinger principle appropriate to our problem. Finally, we introduce the calculus of [17] and describe the behavior of the Birman-Schwinger operator along the spectral minimum of the unperturbed elasticity operator.

The main proof of the accumulation rate asymptotics can be found in $\$ 7$. The general idea of the proof is to obtain properties of the spectrum of the unbounded elasticity operator by investigating the compact Birman-Schwinger operator. This is done by extracting the part of the Birman-Schwinger operator that is responsible for the leading term in the eigenvalue asymptotics, calculating its properties, and estimating the remainder terms. 
1.2. Acknowledgments. The first author is very grateful to A. Laptev and R. Frank for valuable discussions. He acknowledges gratefully the kind hospitality of the KTH during winter visits in 2005 and 2006. Financial support by the Cusanuswerk, the DAAD PPP-programmes, the ENIGMA network and by the DFG grant We 1964/2-1 are gratefully acknowledged.

Moreover, the authors express their gratitude to the referee, the editor T. Suslina, and to V. Sloushch for numerous valuable comments improving the text considerably.

\section{§2. The linear elastostatic operator for zero Poisson coefficient}

2.1. The unperturbed operator. We recall that $G=\mathbb{R}^{2} \times J$ with $J=\left(-\frac{\pi}{2}, \frac{\pi}{2}\right)$. In general, the quadratic form describing linear elastostatics with stress-free boundary conditions is given by

$$
a[u]=\int_{G}\langle\sigma(u), \epsilon(u)\rangle_{\mathbb{C}^{3 \times 3}} \mathrm{~d} x
$$

on all functions $u \in D[a]=H^{1}\left(G ; \mathbb{C}^{3}\right)$. Here

$$
\epsilon(u)=\frac{1}{2}\left(\nabla u+(\nabla u)^{T}\right) \quad \text { and } \quad \sigma(u)=\lambda(\operatorname{Tr} \epsilon(u)) \mathrm{I}+2 \mu \epsilon(u)
$$

denote the strain and stress matrix with the Lamé constants

$$
\mu=\frac{E}{2(1+\nu)} \quad \text { and } \quad \lambda=\frac{E \nu}{(1+\nu)(1-2 \nu)},
$$

where $\nu$ is Poisson's ratio and $E$ is Young's modulus.

In this paper we focus on the special case where Poisson's ratio is zero. For a suitable set of units such that $E=2$, the form (2.1) turns into

$$
a[u]=2 \int_{G}\langle\epsilon(u), \epsilon(u)\rangle_{\mathbb{C}^{3 \times 3}} \mathrm{~d} x \quad \text { on } \quad D[a]=H^{1}\left(G ; \mathbb{C}^{3}\right) .
$$

This form is well defined because, obviously,

$$
a[u] \leq 2\|u\|_{H^{1}\left(G ; \mathbb{C}^{3}\right)}^{2}, \quad u \in H^{1}\left(G ; \mathbb{C}^{3}\right) .
$$

On the other hand, Korn's inequality implies also the reverse estimate

$$
a[u]+\|u\|_{L^{2}\left(G ; \mathbb{C}^{3}\right)}^{2} \geq c\|u\|_{H^{1}\left(G ; \mathbb{C}^{3}\right)}^{2}, \quad u \in H^{1}\left(G ; \mathbb{C}^{3}\right),
$$

for some $c>0$, see 22]. Thus, the nonnegative form $a$ is closed on the maximal domain $D[a]=H^{1}\left(G ; \mathbb{C}^{3}\right)$. It is associated with the positive selfadjoint operator

$$
A=-(\Delta+\operatorname{grad} \operatorname{div})
$$

on the domain

$$
D(A)=\left\{u \in H^{2}\left(G ; \mathbb{C}^{3}\right): \partial_{j} u_{3}+\partial_{3} u_{j}=0, j=1,2,3 \text { on } \partial G\right\} .
$$

2.2. Perturbation by a hole. Let $\Omega_{0} \subset \mathbb{R}^{2}$ be a nonempty, connected, bounded Lipschitz domain. Put $\Omega:=\Omega_{0} \times J$ and $\Omega^{c}=G \backslash \bar{\Omega}$. We shall study the two quadratic forms

$$
\begin{aligned}
a_{\Omega^{c}}[u] & =2 \int_{\Omega^{c}}\langle\epsilon(u), \epsilon(u)\rangle_{\mathbb{C}^{3 \times 3}} \mathrm{~d} x, \quad u \in D\left[a_{\Omega^{c}}\right]:=H^{1}\left(\Omega^{c} ; \mathbb{C}^{3}\right), \\
a_{\Omega}[u] & =2 \int_{\Omega}\langle\epsilon(u), \epsilon(u)\rangle_{\mathbb{C}^{3 \times 3}} \mathrm{~d} x, \quad u \in D\left[a_{\Omega}\right]:=H^{1}\left(\Omega ; \mathbb{C}^{3}\right) .
\end{aligned}
$$

As before, we have

$$
a_{\Omega^{c}}[u] \leq 2\|u\|_{H^{1}\left(\Omega^{c} ; \mathbb{C}^{3}\right)}^{2} \quad \text { and } \quad a_{\Omega}[u] \leq 2\|u\|_{H^{1}\left(\Omega ; \mathbb{C}^{3}\right)}^{2},
$$


and Korn's inequality implies that

$$
\begin{aligned}
a_{\Omega^{c}}[u]+\|u\|_{L^{2}\left(\Omega^{c} ; \mathbb{C}^{3}\right)}^{2} \geq c\left(\Omega^{c}\right)\|u\|_{H^{1}\left(\Omega^{c} ; \mathbb{C}^{3}\right)}^{2}, & & u \in H^{1}\left(\Omega^{c} ; \mathbb{C}^{3}\right), \\
a_{\Omega}[u]+\|u\|_{L^{2}\left(\Omega ; \mathbb{C}^{3}\right)}^{2} \geq c(\Omega)\|u\|_{H^{1}\left(\Omega ; \mathbb{C}^{3}\right)}^{2}, & & u \in H^{1}\left(\Omega ; \mathbb{C}^{3}\right) .
\end{aligned}
$$

Hence, the two forms $a_{\Omega^{c}}$ and $a_{\Omega}$ are closed on the maximal domains $D\left[a_{\Omega^{c}}\right]=H^{1}\left(\Omega^{c} ; \mathbb{C}^{3}\right)$ and $D\left[a_{\Omega}\right]=H^{1}\left(\Omega ; \mathbb{C}^{3}\right)$, respectively. They are associated with the selfadjoint operators $A_{\Omega^{c}}$ in $L^{2}\left(\Omega^{c} ; \mathbb{C}^{3}\right)$ and $A_{\Omega}$ in $L^{2}\left(\Omega ; \mathbb{C}^{3}\right)$, subject to the stress-free boundary conditions

$$
\left(\nabla u+(\nabla u)^{T}\right) \mathbf{n}_{\partial \Omega^{c}}=0, \quad\left(\nabla u+(\nabla u)^{T}\right) \mathbf{n}_{\partial \Omega}=0,
$$

where $\mathbf{n}_{\partial \Omega^{c}}$ and $\mathbf{n}_{\partial \Omega}$ denote the outward unit normals for $\partial \Omega^{c}$ and $\partial \Omega$, respectively. The operator $A_{\Omega}$ describes the interior problem in $\Omega$ and $A_{\Omega^{c}}$ describes the exterior problem in $\Omega^{c}$. The orthogonal sum of these two operators gives

$$
A_{\Gamma}:=A_{\Omega^{c}} \oplus A_{\Omega} \quad \text { in } \quad L^{2}\left(G ; \mathbb{C}^{3}\right)=L^{2}\left(\Omega^{c} ; \mathbb{C}^{3}\right) \oplus L^{2}\left(\Omega ; \mathbb{C}^{3}\right) .
$$

Here we use the notation

$$
\Gamma=\Gamma_{0} \times J \quad \text { with } \quad \Gamma_{0}=\partial \Omega_{0} .
$$

Observe that the operator $A_{\Gamma}$ acts in the same Hilbert space $L^{2}\left(G ; \mathbb{C}^{3}\right)$ as $A$. It is associated with the quadratic form

$$
a_{\Gamma}[u]=2 \int_{G \backslash \Gamma}\langle\epsilon(u), \epsilon(u)\rangle_{\mathbb{C}^{3 \times 3}} \mathrm{~d} x, \quad u \in D\left[a_{\Gamma}\right]:=H^{1}\left(G \backslash \Gamma ; \mathbb{C}^{3}\right) .
$$

We point out that the operator $A_{\Gamma}$ corresponds to a perturbation of $A$ by Neumanntype boundary conditions at $\Gamma$. We have

$$
\begin{array}{r}
D\left(A_{\Gamma}\right)=\left\{u \in H^{2}\left(G \backslash \Gamma ; \mathbb{C}^{3}\right):\left(\nabla u+(\nabla u)^{T}\right) \mathbf{n}_{\partial G}=0 \text { on } \partial G,\right. \\
\left.\left(\nabla u+(\nabla u)^{T}\right) \mathbf{n}_{\Gamma}=0 \text { on } \Gamma\right\},
\end{array}
$$

where $\mathbf{n}_{\partial G}$ and $\mathbf{n}_{\Gamma}$ denote the outward unit normals for $\partial G$ and $\Gamma$, respectively.

\section{§3. Symmetry DECOMPOSITION AND INITIAL SPECTRAL ANALYSIS}

3.1. Spatial and internal symmetries. As in [15], we define the subspaces $\mathcal{H}_{j}, j=$ 1,2 , of $\mathcal{H}=L^{2}\left(G ; \mathbb{C}^{3}\right)$ by

$$
\begin{array}{r}
\mathcal{H}_{j}:=\left\{u \in \mathcal{H}: u_{l}\left(\cdot, \cdot,-x_{3}\right)=(-1)^{j-1} u_{l}\left(\cdot, \cdot, x_{3}\right), l=1,2,\right. \\
\left.u_{3}\left(\cdot, \cdot,-x_{3}\right)=(-1)^{j} u_{3}\left(\cdot, \cdot, x_{3}\right), x_{3} \in J\right\} .
\end{array}
$$

Furthermore, we set

$$
\mathcal{H}_{3}:=\left\{u \in \mathcal{H}: u(x)=\left(u_{1}\left(x_{1}, x_{2}\right), u_{2}\left(x_{1}, x_{2}\right), 0\right), x \in G\right\}
$$

and denote by $\mathcal{H}_{4}$ the orthogonal complement of $\mathcal{H}_{3}$ in $\mathcal{H}_{1}$, which consists of all functions $u \in \mathcal{H}_{1}$ such that

$$
\int_{J} u_{i}\left(x_{1}, x_{2}, x_{3}\right) \mathrm{d} x_{3}=0 \quad \text { for almost every } \quad\left(x_{1}, x_{2}\right) \in \mathbb{R}^{2}, \quad i=1,2 .
$$

By $P_{j}$ we denote the orthogonal projections onto $\mathcal{H}_{j}, j=1, \ldots, 4$. Then $P_{j} P_{1}=P_{1} P_{j}=$ $P_{j}$ for $j=3,4$. Defining

$$
D\left[a^{(j)}\right]:=P_{j} D[a] \subset D[a], \quad j=1, \ldots, 4,
$$

we see that

$$
a[u, v]=0 \quad \text { for all } \quad u \in D\left[a^{(l)}\right], v \in D\left[a^{(j)}\right]
$$


with $l, j=2,3,4$ and $l \neq j$. Hence, these subspaces are reducing 2 for the operator $A$, and $A=A^{(3)} \oplus A^{(4)} \oplus A^{(2)}$ on

$$
\mathcal{H}=\mathcal{H}_{3} \oplus \mathcal{H}_{4} \oplus \mathcal{H}_{2},
$$

where the operators $A^{(j)}$ are the restrictions of $A$ to $D\left(A^{(j)}\right)=D(A) \cap \mathcal{H}_{j}$ and correspond to the closed forms $a^{(j)}$ given by the differential expression (1.1) on $D\left[a^{(j)}\right], j=2,3,4$.

Since the boundary $\Gamma$ is parallel to the $x_{3}$-direction, a simple calculation shows that the orthogonal decomposition (3.3) is also reducing for the perturbed operator $A_{\Gamma}=$ $A_{\Gamma}^{(3)} \oplus A_{\Gamma}^{(4)} \oplus A_{\Gamma}^{(2)}$. In the case of a Lipschitz domain $\Omega=\Omega_{0} \times J$, we have a similar symmetry decomposition for

$$
A_{\Omega}=A_{\Omega}^{(3)} \oplus A_{\Omega}^{(4)} \oplus A_{\Omega}^{(2)} \quad \text { and } \quad A_{\Omega^{c}}=A_{\Omega^{c}}^{(3)} \oplus A_{\Omega^{c}}^{(4)} \oplus A_{\Omega^{c}}^{(2)} .
$$

3.2. Separation of variables for the operator $A$. Applying the unitary Fourier transformation $\Phi$ in $\left(x_{1}, x_{2}\right)$-direction and its inverse $\Phi^{*}$, we see that $\Phi A \Phi^{*}$ admits the orthogonal expansion

$$
\Phi A \Phi^{*}=\int_{\mathbb{R}^{2}}^{\oplus} A(\xi) \mathrm{d} \xi \quad \text { on } \quad \mathcal{H}=\int_{\mathbb{R}^{2}}^{\oplus} h \mathrm{~d} \xi, \quad h=L^{2}\left(J, \mathbb{C}^{3}\right) .
$$

The selfadjoint operators $A(\xi)$ are given by the differential expressions

$$
A(\xi)=\left(\begin{array}{ccc}
-\partial_{3}^{2}+|\xi|^{2}+\xi_{1}^{2} & \xi_{1} \xi_{2} & -i \xi_{1} \partial_{3} \\
\xi_{1} \xi_{2} & -\partial_{3}^{2}+|\xi|^{2}+\xi_{2}^{2} & -i \xi_{2} \partial_{3} \\
-i \xi_{1} \partial_{3} & -i \xi_{2} \partial_{3} & -2 \partial_{3}^{2}+|\xi|^{2}
\end{array}\right)
$$

on the domains

$$
\begin{aligned}
D(A(\xi))=\left\{u \in H^{2}\left(J ; \mathbb{C}^{3}\right): \partial_{3} u_{3}\left( \pm \frac{\pi}{2}\right)=\right. & \partial_{3} u_{1}\left( \pm \frac{\pi}{2}\right)+i \xi_{1} u_{3}\left( \pm \frac{\pi}{2}\right)=0 \\
& \left.\partial_{3} u_{2}\left( \pm \frac{\pi}{2}\right)+i \xi_{2} u_{3}\left( \pm \frac{\pi}{2}\right)=0\right\}
\end{aligned}
$$

The symmetry (3.3) extends to the operators $A(\xi)$. Indeed, if for $j=1,2$ we put

$$
h_{j}:=\left\{u \in h: u_{l}(t)=(-1)^{j-1} u_{l}(-t), l=1,2 ; u_{3}(t)=(-1)^{j} u_{3}(-t)\right\}
$$

and

$$
h_{3}:=\operatorname{span}\{(1,0,0),(0,1,0)\}, \quad h_{4}:=\left\{u \in h_{1}: \int_{J} u_{i}(t) \mathrm{d} t=0, i=1,2\right\}
$$

then

$$
\Phi A^{(j)} \Phi^{*}=\int_{\mathbb{R}^{2}}^{\oplus} A^{(j)}(\xi) \mathrm{d} \xi \quad \text { on } \quad \mathcal{H}_{j}=\int_{\mathbb{R}^{2}}^{\oplus} h_{j} \mathrm{~d} \xi, \quad j=1, \ldots, 4,
$$

where the operators $A^{(j)}(\xi)$ are the restrictions of $A(\xi)$ to $D\left(A^{(j)}(\xi)\right)=D(A(\xi)) \cap h_{j}$. Moreover,

$$
\begin{array}{rll}
A(\xi)=A^{(1)}(\xi) \oplus A^{(2)}(\xi) & \text { on } & h=h_{1} \oplus h_{2}, \\
A(\xi)=A^{(3)}(\xi) \oplus A^{(4)}(\xi) \oplus A^{(2)}(\xi) & \text { on } & h=h_{3} \oplus h_{4} \oplus h_{2} .
\end{array}
$$

\footnotetext{
${ }^{2}$ For $\mathcal{H}_{3}$ and $\mathcal{H}_{4}$ to be reducing it is crucial to work in the case of the zero Poisson coefficient.
} 
3.3. Spectral analysis for the operator $A^{(4)}(\xi)$. Throughout this paper, the spectral resolution of the operator $A^{(4)}$ will be of particular interest. Because of the expansion (3.6), in fact we need to carry out spectral analysis for the operators $A^{(4)}(\xi)$.

Being the restrictions of the nonnegative second order Sturm-Liouville systems (3.4) to $D(A(\xi)) \cap h_{4}$, the operators $A^{(4)}(\xi)$ have a nonnegative discrete spectrum, which accumulates to infinity only. We denote the eigenvalues by $\lambda_{j}(\xi), j \in \mathbb{N}$, where the numbering is in the nondecreasing order, including multiplicities. Note that the eigenvalues depend on the absolute value of $\xi$ only. Thus, sometimes we use the notation $\lambda_{k}=\lambda_{k}(r)$ for $r:=|\xi| \geq 0$.

The following results on the lowest branch of eigenvalues were obtained in [14, 15]. They are crucial for our further investigations. We have

$$
\Lambda:=\min _{\xi \in \mathbb{R}^{2}} \lambda_{1}(\xi)>0
$$

where the minimum is attained on the set $\left\{\xi \in \mathbb{R}^{2}:|\xi|=\varkappa\right\}$ for some $\varkappa>0$. Furthermore, in a suitable neighborhood of this set we have $\lambda_{2}(\xi)>\lambda_{1}(\xi)$, and $\lambda_{1}=\lambda_{1}(r)$ is real analytic. The behavior of $\lambda_{1}(\cdot)$ near its minimum is given by

$$
\lambda_{1}(\varkappa+\varepsilon)=\Lambda+q^{2} \varepsilon^{2}+O\left(\varepsilon^{3}\right) \quad \text { as } \quad \varepsilon \rightarrow 0
$$

with a certain $q>0$. As stated in [15], a numerical evaluation gives

$$
\begin{aligned}
\varkappa & =0.632138 \pm 10^{-6} \\
\Lambda & =1.887837 \pm 10^{-6} \\
q & =0.849748 \pm 10^{-6} .
\end{aligned}
$$

Furthermore, for $\xi \in \mathbb{R}^{2}$ in a neighborhood of $|\xi|=\varkappa$ the eigenfunction corresponding to $\lambda_{1}(\xi)$ is given by $\psi_{1}=\widetilde{\psi}_{1} /\left\|\widetilde{\psi}_{1}\right\|_{L^{2}\left(J ; \mathbb{C}^{3}\right)}$, where

$$
\widetilde{\psi}_{1}\left(\xi, x_{3}\right):=\left(i \xi_{1} d_{1}\left(|\xi|, x_{3}\right), i \xi_{2} d_{1}\left(|\xi|, x_{3}\right), d_{2}\left(|\xi|, x_{3}\right)\right)
$$

and

$$
\begin{aligned}
& d_{1}(r, t):=r \beta \cos \left(\frac{\pi}{2} \beta\right) \cos (\gamma t)+\frac{\gamma^{2} \beta}{r} \cos \left(\frac{\pi}{2} \gamma\right) \cos (\beta t) \\
& d_{2}(r, t):=-r \beta \gamma \cos \left(\frac{\pi}{2} \beta\right) \sin (\gamma t)+r \gamma^{2} \cos \left(\frac{\pi}{2} \gamma\right) \sin (\beta t)
\end{aligned}
$$

with $\beta=\sqrt{\lambda_{1}(r)-r^{2}}$ and $\gamma=\sqrt{\frac{\lambda_{1}(r)}{2}-r^{2}}$. For $\xi$ with $|\xi|=\varkappa$, the functions

$$
w_{\xi}(x)=\psi_{1}\left(\xi, x_{3}\right) \mathrm{e}^{i \xi \cdot\left(\begin{array}{l}
x_{1} \\
x_{2}
\end{array}\right)}
$$

satisfy

$$
-(\Delta+\operatorname{grad} \operatorname{div}) w_{\xi}(x)=\Lambda w_{\xi}(x) \quad \text { for } \quad x \in G .
$$

3.4. The spectrum of $A_{\Omega}$ and $A_{\Omega}^{(4)}$. For a bounded Lipschitz domain $\Omega_{0}$ and the respective hole $\Omega=\Omega_{0} \times J$, the bounds (2.8) and (2.10) show that the quadratic form $a_{\Omega}[u]+\|u\|_{L^{2}\left(\Omega ; \mathbb{C}^{3}\right)}^{2}$ is equivalent to the square of the norm on $H^{1}\left(\Omega ; \mathbb{C}^{3}\right)$. Moreover, under these conditions the embedding of $H^{1}\left(\Omega ; \mathbb{C}^{3}\right)$ into $L^{2}\left(\Omega ; \mathbb{C}^{3}\right)$ is compact. Hence, the spectrum of $A_{\Omega}$ is discrete and accumulates to infinity only. The same holds true for the reduced component $A_{\Omega}^{(4)}$. 
3.5. The essential spectrum. By (3.6), the spectrum of $A^{(4)}$ is given by the union of the eigenvalues $\lambda_{j}(\xi)$ of $A^{(4)}(\xi)$ over all $\xi \in \mathbb{R}^{2}$ and $j \in \mathbb{N}$. The eigenvalues are analytic with respect to $|\xi|$. Moreover, Korn's inequality shows that $\lambda_{j}(\xi)+d \geq c|\xi|^{2}$ for suitable constants $c, d>0$. Therefore, the spectrum of $A^{(4)}$ is pure absolutely continuous. Since

$$
\min \sigma_{e}\left(A^{(4)}\right)=\min _{\xi, j} \lambda_{j}(\xi)=\min _{\xi} \lambda_{1}(\xi)=\Lambda,
$$

the essential spectrum $\sigma_{e}\left(A^{(4)}\right)$ coincides with $[\Lambda, \infty)$.

As in [23], it can be shown that the essential spectrum of the locally perturbed operator $A_{\Gamma}^{(4)}$ coincides with the essential spectrum of $A^{(4)}$. Moreover, recall that $A_{\Gamma}=A_{\Omega^{c}} \oplus A_{\Omega}$, so that $A_{\Gamma}^{(4)}=A_{\Omega^{c}}^{(4)} \oplus A_{\Omega}^{(4)}$. Since the spectrum of $A_{\Omega}^{(4)}$ is purely discrete, the essential spectrum of $A_{\Omega^{c}}^{(4)}$ coincides with the essential spectrum of $A_{\Gamma}^{(4)}$, and we conclude finally that

$$
\sigma_{e}\left(A_{\Omega^{c}}^{(4)}\right)=\sigma_{e}\left(A_{\Gamma}^{(4)}\right)=\sigma_{e}\left(A^{(4)}\right)=[\Lambda, \infty) .
$$

Moreover, we shall see that the perturbed operators $A_{\Omega^{c}}^{(4)}$ and $A_{\Gamma}^{(4)}$ have infinite discrete spectrum on $[0, \Lambda)$. Since a similar analysis for the full operators yields

$$
\sigma_{e}\left(A_{\Omega^{c}}\right)=\sigma_{e}\left(A_{\Gamma}\right)=\sigma_{e}(A)=[0, \infty),
$$

this discrete spectrum gives rise to embedded eigenvalues of $A_{\Omega^{c}}$ and $A_{\Gamma}$.

The reduction of the problem to that on the subspace $\mathcal{H}_{4}$ is a crucial step, which "uncovers" these eigenvalues and opens them for a detailed analysis via variational techniques.

\section{$\S 4$. Statement of the main Results}

4.1. Existence of infinitely many eigenvalues. In the following, we state our main results on the existence and accumulation behavior of the eigenvalues of $A_{\Omega^{c}}^{(4)}$ and $A_{\Gamma}^{(4)}$.

Theorem 4.1. The operators $A_{\Omega^{c}}^{(4)}$ and $A_{\Gamma}^{(4)}$ have infinitely many eigenvalues in $[0, \Lambda)$.

Since the spectrum of $A_{\Omega^{c}}^{(4)}$ and $A_{\Gamma}^{(4)}$ in $[0, \Lambda)$ is discrete, these eigenvalues will accumulate to the threshold $\Lambda$ only.

Here we provide a short proof, which involves a result in [15].

Proof. Let $\Gamma=\partial \Omega_{0} \times J$ be the Lipschitz boundary of the hole $\Omega=\Omega_{0} \times J$. Also, let $A_{\alpha}^{(4)}$ be the operator associated with the quadratic form

$$
a_{\alpha}^{(4)}[u]=2 \int_{G}\left(1-\alpha \chi_{\Omega}\right)\langle\epsilon(u), \epsilon(u)\rangle_{\mathbb{C}^{3 \times 3}} \mathrm{~d} x, \quad u \in D\left[a_{\alpha}^{(4)}\right]:=H^{1}\left(G ; \mathbb{C}^{3}\right) \cap \mathcal{H}_{4},
$$

and let $A_{\alpha, \Gamma}^{(4)}$ be the operator associated with the form

$$
a_{\alpha, \Gamma}^{(4)}[u]=2 \int_{G \backslash \Gamma}\left(1-\alpha \chi_{\Omega}\right)\langle\epsilon(u), \epsilon(u)\rangle_{\mathbb{C}^{3 \times 3}} \mathrm{~d} x, \quad u \in D\left[a_{\alpha, \Gamma}^{(4)}\right],
$$

where $D\left[a_{\alpha, \Gamma}^{(4)}\right]:=H^{1}\left(G \backslash \Gamma ; \mathbb{C}^{3}\right) \cap \mathcal{H}_{4}$. Note that $A_{\alpha, \Gamma}^{(4)}$ can be viewed as a perturbation of $A_{\alpha}^{(4)}$ by Neumann-type boundary conditions on $\Gamma$. Thus, we have $A_{\alpha, \Gamma}^{(4)}<A_{\alpha}^{(4)}$. Moreover, by analogy with [23], the essential spectra of the two operators coincide.

It was shown in Theorem 4.1 in 15 that $A_{\alpha}^{(4)}$ has infinitely many eigenvalues in $[0, \Lambda)$ for any $\alpha \in(0,1)$. By a standard variational argument, the operator $A_{\alpha, \Gamma}^{(4)}$ must have infinitely many eigenvalues in $[0, \Lambda)$ as well. Note that

$$
A_{\alpha, \Gamma}^{(4)}=A_{\Omega^{c}}^{(4)} \oplus(1-\alpha) A_{\Omega}^{(4)} .
$$


Since $A_{\Omega}^{(4)}$ has purely discrete spectrum, the spectra of the operators $A_{\Omega^{c}}^{(4)}$ and $A_{\alpha, \Gamma}^{(4)}$ below $\Lambda$ differ only in finitely many eigenvalues. Thus, also $A_{\Omega^{c}}^{(4)}$ has infinitely many eigenvalues in $[0, \Lambda)$.

4.2. Accumulation rate of the eigenvalues near $\Lambda$. For asymptotic estimates on the accumulation of eigenvalues at $\Lambda$, we set

$$
w(t):=t^{-2 t}, \quad t>1 .
$$

For formal convenience we put $\omega(t)=t^{-1}$ for $0<t \leq 1$, extending $w$ to a continuous bijection from $\mathbb{R}_{+}$to $\mathbb{R}_{+}$. Furthermore, for a selfadjoint operator $T$ we denote by $n_{-}(s, T)$ the number of eigenvalues smaller than $s \in \mathbb{R}$ (including multiplicities).

Theorem 4.2. Consider the plate with a hole $\Omega=\Omega_{0} \times J$, and let $\Gamma=\partial \Omega_{0} \times J$. Then

$$
\lim _{\tau \rightarrow 0+} \frac{n_{-}\left(\Lambda-\tau, A_{\Gamma}^{(4)}\right)}{w^{-1}(\tau)}=\lim _{\tau \rightarrow 0+} \frac{n_{-}\left(\Lambda-\tau, A_{\Omega^{c}}^{(4)}\right)}{w^{-1}(\tau)}=1 .
$$

Our result is similar to the asymptotics obtained in [15] for the elastic plate with changes of material properties.

Asymptotical estimates on the eigenvalues can be derived easily from these expressions. Let $\left\{\nu_{k}\right\}_{k \in \mathbb{N}}$ denote the eigenvalues of $A_{\Omega^{c}}^{(4)}$ and $\left\{\widetilde{\nu}_{k}\right\}_{k \in \mathbb{N}}$ the eigenvalues of $A_{\Gamma}^{(4)}$ that lie below $\Lambda$, numbered in the nondecreasing order including multiplicities.

Corollary 4.3. Consider the plate with a hole $\Omega=\Omega_{0} \times J$. Then for every $\varepsilon>0$ there exists $N_{\varepsilon}>0$ such that

$$
w((1+\varepsilon) k) \leq \Lambda-\nu_{k} \leq w((1-\varepsilon) k) \quad \text { for } \quad k>N_{\varepsilon} .
$$

Moreover,

$$
\ln \left(\Lambda-\nu_{k}\right)=-2 k \ln k+o(k \ln k) \quad \text { as } \quad k \rightarrow \infty .
$$

In the remaining part of this paper we prove Theorem 4.2, Note that for the hole $\Omega=\Omega_{0} \times J$ with boundary $\Gamma=\partial \Omega_{0} \times J$ we have

$$
A_{\Gamma}^{(4)}=A_{\Omega^{c}}^{(4)} \oplus A_{\Omega}^{(4)} .
$$

Since $A_{\Omega}^{(4)}$ has purely discrete spectrum, the spectra of $A_{\Gamma}^{(4)}$ and $A_{\Omega^{c}}^{(4)}$ below $\Lambda$ differ only in finitely many eigenvalues. Thus, it suffices to prove Theorem 4.2 for $A_{\Gamma}^{(4)}$ only.

\section{§5. Passage to the Resolvents}

5.1. Inverse operators. It is advantageous to study the geometric perturbation of $A^{(4)}$ to $A_{\Gamma}^{(4)}$ in terms of an additive perturbation of the inverse operators. Since $\sigma\left(A^{(4)}\right)=$ $[\Lambda, \infty)$, the operator $A^{(4)}$ has a bounded inverse $R:=\left(A^{(4)}\right)^{-1}$. Put $R_{\Gamma}:=\left(A_{\Gamma}^{(4)}\right)^{-1}$.

Lemma 5.1. The operator $R_{\Gamma}$ is bounded on $L^{2}\left(G ; \mathbb{C}^{3}\right)$.

Proof. In view of (3.15), it remains to show that 0 is not an eigenvalue of $A_{\Gamma}^{(4)}$. Suppose the contrary. Then the corresponding eigenfunction $u$ satisfies

$$
0=a_{\Gamma}[u]=\int_{G \backslash \Gamma} 2 \sum_{i}\left|\partial_{i} u_{i}\right|^{2}+\sum_{i<j}\left|\partial_{i} u_{j}+\partial_{j} u_{i}\right|^{2} \mathrm{~d} x .
$$

In particular, $\partial_{3} u_{3}=0$ in $G \backslash \Gamma$. Since $u \in \mathcal{H}_{4}, u_{3}$ is antisymmetric, whence $u_{3}=0$. Inserting this into (5.1), we see that $\partial_{3} u_{1}=\partial_{3} u_{2}=0$ in $G \backslash \Gamma$. Again, the condition $u \in \mathcal{H}_{4}$ implies $u_{1}=u_{2}=0$. Thus, $u=0$, which proves that zero is not an eigenvalue of $A_{\Gamma}^{(4)}$. 
Both $R$ and $R_{\Gamma}$ are positive operators, and we denote the associated quadratic forms by $r$ and $r_{\Gamma}$, respectively. Since $D\left[a^{(4)}\right] \subset D\left[a_{\Gamma}^{(4)}\right]$, it follows that $A_{\Gamma}^{(4)}<A^{(4)}$ in the quadratic form sense. Therefore, the operator

$$
B_{\Gamma}:=R_{\Gamma}-R
$$

is bounded and nonnegative. In fact, $B_{\Gamma}$ is a compact operator; this can be shown by using the standard method of [23]. In the following we give an initial characterization of $B_{\Gamma}$. A more detailed analysis of $B_{\Gamma}$ is provided in Subsection 6.4.

5.2. First analysis of $B_{\Gamma}$. Since $\Gamma_{0}$ is Lipschitz, it has a surface measure $S_{\Gamma_{0}}$ and a unit normal $\mathbf{n}_{\Gamma_{0}}$, which exists $S_{\Gamma_{0}}$-almost everywhere on $\Gamma_{0}$; see [22, p. 96]. For closed curves $\Gamma_{0}$ we choose $\mathbf{n}_{\Gamma_{0}}$ to point into the unbounded part of $\mathbb{R}^{2}$. For $\Gamma=\Gamma_{0} \times J$, the corresponding unit normal is given by

$$
\mathbf{n}_{\Gamma}=\left(\begin{array}{c}
\mathbf{n}_{\Gamma_{0}} \\
0
\end{array}\right) .
$$

Assume the normal vector exists at a point $x \in \Gamma$. We can choose some sufficiently small neighborhood $\mathcal{O} \subset G$ of $x$ such that $\mathcal{O} \backslash \Gamma$ consists of two disjoint sets. We denote them by $\mathcal{O}^{+}$and $\mathcal{O}^{-}$so that $x \pm \varepsilon \mathbf{n}_{\Gamma} \in \mathcal{O}^{ \pm}$for sufficiently small $\varepsilon>0$. Then

$$
\gamma_{D}^{+} u(x):=\lim _{\substack{y \rightarrow x \\ y \in \mathcal{O}^{+}}} u(y) \text { and } \gamma_{D}^{-} u(x):=\lim _{\substack{y \rightarrow x \\ y \in \mathcal{O}^{-}}} u(y)
$$

are the trace operators for $u \in \mathrm{C}_{b}(G \backslash \Gamma)$ with respect to $\Gamma$. The operators $\gamma_{D}^{ \pm}$admit extensions to bounded operators

$$
\gamma_{D}^{ \pm}: H^{s}(G \backslash \Gamma) \rightarrow H^{s-1 / 2}(\Gamma), \quad 1 / 2<s \leq 1
$$

see [22, p. 100].

The application of $\gamma_{D}^{ \pm}$to a vector-valued or matrix-valued function means an individual application to each vector or matrix component. If for a given $u$ we have $\gamma_{D}^{+} u=\gamma_{D}^{-} u$, then we use also the shorthand notation $\gamma_{D} u:=\gamma_{D}^{ \pm} u$.

For functions $u \in \mathrm{C}_{b}^{1}\left(G \backslash \Gamma ; \mathbb{C}^{3}\right)$, we define the conormal derivative with respect to the operator $A$ at $\Gamma$ by

$$
\gamma_{N}^{ \pm} u:=2^{-\frac{1}{2}}\left(\gamma_{D}^{ \pm}\left(\nabla u+(\nabla u)^{T}\right)\right) \mathbf{n}_{\Gamma} \quad \text { on } \quad \Gamma .
$$

Again we set $\gamma_{N} u:=\gamma_{N}^{+} u$ if $\gamma_{N}^{+} u=\gamma_{N}^{-} u$. The boundedness of the operators (5.5) implies that the $\gamma_{N}^{ \pm}$extend to bounded operators

$$
\gamma_{N}^{ \pm}: H^{s}\left(G \backslash \Gamma ; \mathbb{C}^{3}\right) \rightarrow H^{s-3 / 2}\left(\Gamma ; \mathbb{C}^{3}\right), \quad 3 / 2<s \leq 2 .
$$

Lemma 5.2. For any $v \in \mathcal{H}_{4}$, the function $B_{\Gamma} v$ is a unique solution of the boundaryvalue problem

$$
-(\Delta+\operatorname{grad} \operatorname{div}) u=0 \quad \text { in } \quad G \backslash \Gamma, \quad \gamma_{N} u=-\gamma_{N} R v \quad \text { on } \quad \Gamma,
$$

for $u \in \mathcal{H}_{4}$ with $\left(\nabla u+(\nabla u)^{T}\right) \mathbf{n}_{\partial G}=0$ on $\partial G$.

Proof. By the definition of $B_{\Gamma}$ in (5.2), we have

$$
B_{\Gamma} v=R_{\Gamma} v-R v \in D\left(A_{\Gamma}^{(4)}\right)-D\left(A^{(4)}\right) \subset \mathcal{H}_{4} .
$$

Furthermore,

$$
-(\Delta+\operatorname{grad} \operatorname{div}) B_{\Gamma} v=v-v=0 \quad \text { in } \quad G \backslash \Gamma
$$

and

$$
\gamma_{N} B_{\Gamma} v=\gamma_{N} R_{\Gamma} v-\gamma_{N} R v=-\gamma_{N} R v .
$$

It remains to show that the solution of the problem (5.7) is unique. Suppose that $u \in \mathcal{H}_{4}$ with $\left(\nabla u+(\nabla u)^{T}\right) \mathbf{n}_{\partial G}=0$ on $\partial G$ satisfies (5.7). Put $w:=u+R v$. Then 
$w \in P_{4} H^{2}\left(G \backslash \Gamma ; \mathbb{C}^{3}\right),\left(\nabla w+(\nabla w)^{T}\right) \mathbf{n}_{\partial G}=0$ on $\partial G$ and $\gamma_{N} w=0$ on $\Gamma$. Thus, $w \in D\left(A_{\Gamma}^{(4)}\right)$. Since

$$
A_{\Gamma}^{(4)} w=-(\Delta+\operatorname{grad} \operatorname{div}) w=0-(\Delta+\operatorname{grad} \operatorname{div}) R v=v,
$$

we see that $w=R_{\Gamma} v$. This proves that $u=B_{\Gamma} v$.

\section{§6. Auxiliary material}

6.1. The classes $\Sigma_{\pi}$ and $\mathcal{S}^{\pi}$. In order to describe the accumulation rate for the eigenvalues of $A_{\Gamma}^{(4)}$ at $\Lambda$, we need to generalize the well-known weak Neumann-Schatten classes $\Sigma_{p}$; see [24, 11.6], and the classes $\mathcal{S}^{p}$ introduced by Safronov [25, 17]. This is done as in 15 .

Let $\pi: \mathbb{R}_{+} \rightarrow \mathbb{R}_{+}$be a function with $\lim _{s \rightarrow 0+} \pi(s)=0$. For suitable compact operators $T$, we define the functionals

$$
\Delta_{\pi}(T)=\limsup _{s \rightarrow 0+} \pi(s) n(s, T), \quad \delta_{\pi}(T)=\liminf _{s \rightarrow 0+} \pi(s) n(s, T) .
$$

Here $n(s, T)$ denotes the number of singular values of $T$ strictly larger than $s$ (including multiplicities). We denote by $\Sigma_{\pi}$ the class of compact operators $T$ such that $\Delta_{\pi}(T)<\infty$. Similarly, for suitable operator-valued functions $\mathcal{T}: \mathbb{R}_{+} \rightarrow \mathfrak{S}_{\infty}$ we define the functionals

$$
\Delta_{\pi}(\mathcal{T})=\limsup _{s \rightarrow 0+} \pi(s) n(1, \mathcal{T}(s)), \quad \delta_{\pi}(\mathcal{T})=\liminf _{s \rightarrow 0+} \pi(s) n(1, \mathcal{T}(s))
$$

We denote by $\mathcal{S}^{\pi}$ the class of operator-valued functions $\mathcal{T}$ such that $\Delta_{\pi}(\beta \mathcal{T})<\infty$ for all $\beta>0$. Furthermore, $\mathcal{S}_{0}^{\pi}$ is the class of all $\mathcal{T} \in \mathcal{S}^{\pi}$ such that $\Delta_{\pi}(\beta \mathcal{T})=0$ for all $\beta>0$. We will need the following two lemmas, which were proved in [15]. See also [17] for the polynomial case.

Lemma 6.1. For $\mathcal{T} \in \mathcal{S}^{\pi}$ and $\mathcal{T}_{0} \in \mathcal{S}_{0}^{\pi}$,

$$
\begin{aligned}
& \lim _{\beta \rightarrow 1} \Delta_{\pi}(\beta \mathcal{T})=\Delta_{\pi}(\mathcal{T}) \quad \text { implies } \quad \Delta_{\pi}\left(\mathcal{T}+\mathcal{T}_{0}\right)=\Delta_{\pi}(\mathcal{T}), \quad \text { and } \\
& \lim _{\beta \rightarrow 1} \delta_{\pi}(\beta \mathcal{T})=\delta_{\pi}(\mathcal{T}) \quad \text { implies } \quad \delta_{\pi}\left(\mathcal{T}+\mathcal{T}_{0}\right)=\delta_{\pi}(\mathcal{T}) .
\end{aligned}
$$

Lemma 6.2. Let $\mathcal{T}: \mathbb{R}_{+} \rightarrow \mathfrak{S}_{\infty}$, and let $\mathcal{T}(s) \rightarrow T$ in $\mathfrak{S}_{\infty}$ as $s \rightarrow 0$. Then $\mathcal{T} \in \mathcal{S}_{0}^{\pi}$ for all $\pi: \mathbb{R}_{+} \rightarrow \mathbb{R}_{+}$with $\lim _{s \rightarrow 0+} \pi(s)=0$.

6.2. A modified Birman-Schwinger principle. Using the definitions in 95 , we define the Birman-Schwinger operator

$$
\mathcal{Y}(\tau):=\left(\frac{1}{\Lambda-\tau}-R\right)^{-\frac{1}{2}} B_{\Gamma}\left(\frac{1}{\Lambda-\tau}-R\right)^{-\frac{1}{2}}
$$

in $\mathcal{H}_{4}$ for $\tau \in(0, \Lambda)$.

For an operator $T$, we denote by $n_{+}(s, T)$ and $n_{-}(s, T)$ the number of eigenvalues strictly larger and strictly smaller than $s>0$, respectively (counting multiplicities).

We shall need the following version of the Birman-Schwinger principle [28, 29].

Lemma 6.3. Let $\tau \in(0, \Lambda)$. Then

$$
n_{-}\left(\Lambda-\tau, A_{\Gamma}^{(4)}\right)=n_{+}(1, \mathcal{Y}(\tau)) .
$$


Proof. Using Glazman's lemma [24, 10.2.2], we obtain

$$
\begin{aligned}
& n_{-}\left(\Lambda-\tau, A_{\Gamma}^{(4)}\right)=n_{+}\left((\Lambda-\tau)^{-1}, R+B_{\Gamma}\right) \\
& \quad=\sup _{L}\left\{\operatorname{dim} L:(\Lambda-\tau)^{-1}\|u\|^{2}<r[u]+\left\|B_{\Gamma}^{\frac{1}{2}} u\right\|^{2}, \quad 0 \neq u \in L\right\} \\
& \quad=\sup _{L}\left\{\operatorname{dim} L:\|v\|^{2}<\left\|B_{\Gamma}^{\frac{1}{2}}\left((\Lambda-\tau)^{-1}-R\right)^{-\frac{1}{2}} v\right\|^{2}, \quad 0 \neq v \in L\right\} \\
& \quad=n_{+}(1, \mathcal{Y}(\tau)),
\end{aligned}
$$

where $L$ runs through subspaces of $\mathcal{H}_{4}$.

6.3. Reduction to the spectral minimum of $A^{(4)}$. The reduction of $A^{(4)}$ to its spectral minimum was already described in [15]. Nevertheless, here we present it again because of its importance for the paper. As in Subsection [3.2, $\lambda_{1}(\xi)$ denotes the lowest branch of eigenvalues of $A^{(4)}(\xi)$. The corresponding normed eigenfunction in $L^{2}\left(J ; \mathbb{C}^{3}\right)$ is $\psi_{1}(\xi, \cdot)$. We set

$$
\lambda_{0}(\xi):=\lambda_{1}(\xi)-\Lambda \text { for } \xi \in \Xi,
$$

where $\Xi:=\lambda_{1}^{-1}([\Lambda, \Lambda+\delta))$ for suitable $\delta>0$. The spectral projection of $A^{(4)}$ onto $[\Lambda, \Lambda+\delta)$ is given by

$$
\left(\Pi_{c} u\right)(x):=\Phi_{\xi \rightarrow\left(\begin{array}{l}
x_{1} \\
x_{2}
\end{array}\right)}^{*}\left(\chi_{\Xi}(\xi)\left\langle(\Phi u)(\xi, \cdot), \psi_{1}(\xi, \cdot)\right\rangle_{L^{2}\left(J ; \mathbb{C}^{3}\right)} \psi_{1}\left(\xi, x_{3}\right)\right), u \in \mathcal{H}_{4} .
$$

Then the unitary operator

$$
\Pi_{0}: \Pi_{c} \mathcal{H}_{4} \rightarrow L^{2}(\Xi), \quad\left(\Pi_{0} u\right)(\xi):=\left\langle(\Phi u)(\xi, \cdot), \psi_{1}(\xi, \cdot)\right\rangle_{L^{2}\left(J ; \mathbb{C}^{3}\right)}
$$

maps $\Pi_{c} \mathcal{H}_{4}$ into scalar-valued functions.

Next, we define

$$
P(\xi):=\left\{\begin{array}{lll}
+\sqrt{\lambda_{0}(\xi)} & \text { if } & |\xi| \geq \varkappa, \\
-\sqrt{\lambda_{0}(\xi)} & \text { if } & |\xi| \leq \varkappa .
\end{array}\right.
$$

The properties of $\lambda_{1}(\xi)$ collected in Subsection 3.2 show that $P \in \mathrm{C}^{1}(\Xi)$ for sufficiently small $\delta>0$. Furthermore, $\mathrm{P}$ depends on $|\xi|$ only. We have

$$
|\nabla P(\xi)|=q \text { for }|\xi|=\varkappa,
$$

whence $|\nabla P(\xi)|>0$ for $\xi \in \Xi$ if $\delta>0$ is sufficiently small. Now we are able to describe $A^{(4)}$ by a suitable direct integral. Let

$$
M_{\lambda}=\left\{\xi \in \mathbb{R}^{2}: P(\xi)=\lambda\right\} \quad \text { for } \quad \lambda \in \Theta:=(-\sqrt{\delta}, \sqrt{\delta}) .
$$

In particular, $M_{0}=\left\{\xi \in \mathbb{R}^{2}:|\xi|=\varkappa\right\}$ is the set where $\lambda_{1}(\cdot)$ attains its minimum. We denote by $\mathrm{d} M_{\lambda}$ the measure on $M_{\lambda}$ induced by $\mathrm{d} \xi$ on $\mathbb{R}^{2}$. Furthermore, we set $\mathrm{d} \mu_{\lambda}:=|\nabla P|^{-1} \mathrm{~d} M_{\lambda}$ and

$$
G(\lambda):=L^{2}\left(M_{\lambda}, \mathrm{d} \mu_{\lambda}\right) \quad \text { for } \quad \lambda \in \Theta .
$$

The measures $\mathrm{d} \xi$ and $\mathrm{d} \mu_{\lambda} \mathrm{d} \lambda$ coincide on $\Xi$. With these definitions, we have

$$
\lambda_{0}=\int_{\Theta}^{\oplus} \lambda^{2} \mathrm{~d} \lambda \quad \text { on } \quad L^{2}(\Xi)=\int_{\Theta}^{\oplus} G(\lambda) \mathrm{d} \lambda,
$$

where $\lambda_{0}$ denotes the operator of multiplication by $\lambda_{0}(\cdot)$ in $L^{2}(\Xi)$ and $\lambda^{2}$ is the operator of multiplication by $\lambda^{2}$ in $G(\lambda)$. The spaces $G(\lambda)$ are unitarily equivalent. We denote by $U_{\lambda}: G(\lambda) \rightarrow G(0)$ the corresponding unitary operator. It follows that the space $L^{2}(\Xi)$ is equivalent to

$$
G_{\Theta}:=L^{2}(\Theta) \otimes G(0),
$$


where the corresponding unitary operator $\Upsilon: L^{2}(\Xi) \rightarrow G_{\Theta}$ is given by

$$
(\Upsilon u)(\lambda)=U_{\lambda}\left(\left.u\right|_{M_{\lambda}}\right) \quad \text { for } \quad u \in L^{2}(\Xi) \quad \text { and almost every } \quad \lambda \in \Theta .
$$

Finally, we define the isometry

$$
F:=\Upsilon \Pi_{0}
$$

between $\Pi_{c} \mathcal{H}_{4}$ and $G_{\Theta}$, which reduces the operator $A^{(4)}-\Lambda$ to its essential behavior near the spectral minimum. Namely, if we define $J_{\lambda} u:=(F u)(\lambda)$ for $u \in \Pi_{c} \mathcal{H}_{4}$ and almost every $\lambda \in \Theta$, then

$$
J_{\lambda}\left(A^{(4)}-\Lambda\right) u=\lambda^{2} J_{\lambda} u .
$$

6.4. Reduction of $\mathcal{Y}(\tau)$ to the spectral minimum. We reduce the Birman-Schwinger operator by considering its restriction to the wave functions at the spectral minimum of $A^{(4)}$. The main task is to describe the behavior of $B_{\Gamma}$ on the wave functions. Since a direct calculation is only possible for rotation invariant perturbations, we restrict ourselves to the case of a circular boundary perturbation

$$
\Gamma:=\Gamma^{\beta}=\left\{x \in \mathbb{R}^{2}:|x|=\beta\right\} \times J, \quad \beta>0 .
$$

Note that the decomposition of $L^{2}\left(G ; \mathbb{C}^{3}\right)$ into symmetry spaces obtained in Subsection 3.1 carries over to $L^{2}\left(\Gamma^{\beta} ; \mathbb{C}^{3}\right)$. In the following, we denote by $P_{4}$ not only the orthogonal projection onto $\mathcal{H}_{4}$ in $L^{2}\left(G ; \mathbb{C}^{3}\right)$ but also the projection onto the corresponding symmetry space in $L^{2}\left(\Gamma^{\beta} ; \mathbb{C}^{3}\right)$.

The reduction procedure for $\mathcal{Y}(\tau)$ involves operators from elastic potential theory. These operators shall be introduced as follows. First, we mention that

$$
\gamma_{N} R: P_{4} L^{2}\left(G ; \mathbb{C}^{3}\right) \rightarrow P_{4} L^{2}\left(\Gamma^{\beta} ; \mathbb{C}^{3}\right)
$$

is a bounded operator. This follows immediately from the boundedness of the operators $\gamma_{N}: H^{2}\left(G ; \mathbb{C}^{3}\right) \rightarrow H^{\frac{1}{2}}\left(\Gamma^{\beta} ; \mathbb{C}^{3}\right)$ and $R: P_{4} L^{2}\left(G ; \mathbb{C}^{3}\right) \rightarrow P_{4} H^{2}\left(G ; \mathbb{C}^{3}\right)$. The latter follows from the boundedness of $R$ in $P_{4} L^{2}\left(G ; \mathbb{C}^{3}\right)$ and from the estimate

$$
\|A u\|^{2}+\|u\|^{2} \geq c\|u\|_{H^{2}\left(G ; \mathbb{C}^{3}\right)}^{2}, \quad u \in D(A)
$$

for some $c>0$. Note that $(6.8)$ is a consequence of local regularity estimates going back to Nirenberg [30; see also [22, p. 133]. We conclude that the adjoint operator $\left(\gamma_{N} R\right)^{*}: P_{4} L^{2}\left(\Gamma^{\beta} ; \mathbb{C}^{3}\right) \rightarrow P_{4} L^{2}\left(G ; \mathbb{C}^{3}\right)$ is well defined and bounded. This operator is known as the double-layer potential operator. The following properties of $\left(\gamma_{N} R\right)^{*}$ will be of importance for us. We refer to [22, Chapter 6] for proofs and further references.

Lemma 6.4. The double-layer operator $\left(\gamma_{N} R\right)^{*}$ gives rise to bounded linear operators

$$
\begin{aligned}
& \gamma_{D}^{ \pm}\left(\gamma_{N} R\right)^{*}: P_{4} H^{s+1 / 2}\left(\Gamma^{\beta} ; \mathbb{C}^{3}\right) \rightarrow P_{4} H^{s+1 / 2}\left(\Gamma^{\beta} ; \mathbb{C}^{3}\right), \\
& \gamma_{N}\left(\gamma_{N} R\right)^{*}: P_{4} H^{s+1 / 2}\left(\Gamma^{\beta} ; \mathbb{C}^{3}\right) \rightarrow P_{4} H^{s-1 / 2}\left(\Gamma^{\beta} ; \mathbb{C}^{3}\right)
\end{aligned}
$$

for $-\frac{1}{2} \leq s \leq \frac{1}{2}$. Moreover, let $\varphi \in P_{4} L^{2}\left(\Gamma^{\beta} ; \mathbb{C}^{3}\right)$, and let $u:=-\left(\gamma_{N} R\right)^{*} \varphi$. Then

$$
-(\Delta+\operatorname{grad} \operatorname{div}) u=0 \quad \text { on } \quad G \backslash \Gamma^{\beta}
$$

and

$$
0=\gamma_{N}^{-} u-\gamma_{N}^{+} u, \quad \varphi=\gamma_{D}^{-} u-\gamma_{D}^{+} u \quad \text { on } \quad \Gamma^{\beta} .
$$

The above results allow us to define the operator

$$
D \varphi:=-\gamma_{N}\left(\gamma_{N} R\right)^{*} \varphi
$$

for $\varphi \in P_{4} H^{1}\left(\Gamma^{\beta} ; \mathbb{C}^{3}\right)$. The operator $D$ is often called a hypersingular operator, which refers to its representation as an integral operator. The next lemma shows that $D$ is a positive definite selfadjoint operator in $P_{4} L^{2}\left(\Gamma^{\beta} ; \mathbb{C}^{3}\right)$. 
Lemma 6.5. We have

$$
c^{-1}\|\varphi\|_{H^{\frac{1}{2}}\left(\Gamma^{\beta} ; \mathbb{C}^{3}\right)}^{2} \leq\langle D \varphi, \varphi\rangle_{L^{2}\left(\Gamma^{\beta} ; \mathbb{C}^{3}\right)} \leq c\|\varphi\|_{H^{\frac{1}{2}}\left(\Gamma^{\beta} ; \mathbb{C}^{3}\right)}^{2}, \quad \varphi \in P_{4} H^{\frac{1}{2}}\left(\Gamma^{\beta} ; \mathbb{C}^{3}\right),
$$

for some $c>0$.

Proof. Looking at (6.9) in Lemma 6.4, we immediately deduce that

$$
\langle D \varphi, \varphi\rangle_{L^{2}\left(\Gamma^{\beta} ; \mathbb{C}^{3}\right)} \leq\|D \varphi\|_{H^{-\frac{1}{2}}\left(\Gamma^{\beta} ; \mathbb{C}^{3}\right)}\|\varphi\|_{H^{\frac{1}{2}}\left(\Gamma^{\beta} ; \mathbb{C}^{3}\right)} \leq c\|\varphi\|_{H^{\frac{1}{2}}\left(\Gamma^{\beta} ; \mathbb{C}^{3}\right)}^{2}
$$

for all $\varphi \in P_{4} H^{\frac{1}{2}}\left(\Gamma^{\beta} ; \mathbb{C}^{3}\right)$ and some $c>0$. This proves the upper estimate. For the lower estimate, we follow the arguments in [31, 6.6.2]. Let $\varphi \in P_{4} H^{\frac{1}{2}}\left(\Gamma^{\beta} ; \mathbb{C}^{3}\right)$, and let $u=-\left(\gamma_{N} R\right)^{*} \varphi$. Then Lemma 6.4 gives

$$
\begin{aligned}
\langle D \varphi, \varphi\rangle_{L^{2}\left(\Gamma^{\beta} ; \mathbb{C}^{3}\right)} & =\left\langle\gamma_{N} u, \gamma_{D}^{-} u-\gamma_{D}^{+} u\right\rangle_{L^{2}\left(\Gamma^{\beta} ; \mathbb{C}^{3}\right)} \\
& =\left\langle\gamma_{N}^{-} u, \gamma_{D}^{-} u\right\rangle_{L^{2}\left(\Gamma^{\beta} ; \mathbb{C}^{3}\right)}-\left\langle\gamma_{N}^{+} u, \gamma_{D}^{+} u\right\rangle_{L^{2}\left(\Gamma^{\beta} ; \mathbb{C}^{3}\right)} \\
& =2 \int_{G \backslash \Gamma^{\beta}}|\epsilon(u)|_{\mathbb{C}^{3 \times 3}}^{2} \mathrm{~d} x .
\end{aligned}
$$

On the other hand, using Lemma 6.4 again, and recalling the embedding properties of the trace operator (5.5) and Korn's inequality, we see that

$$
\begin{aligned}
\|\varphi\|_{H^{\frac{1}{2}}\left(\Gamma^{\beta} ; \mathbb{C}^{3}\right)}^{2} & =\left\|\gamma_{D}^{-} u-\gamma_{D}^{+} u\right\|_{H^{\frac{1}{2}}\left(\Gamma^{\beta} ; \mathbb{C}^{3}\right)}^{2} \\
& \leq 2\left\|\gamma_{D}^{-} u\right\|_{H^{\frac{1}{2}}\left(\Gamma^{\beta} ; \mathbb{C}^{3}\right)}^{2}+2\left\|\gamma_{D}^{+} u\right\|_{H^{\frac{1}{2}}\left(\Gamma^{\beta} ; \mathbb{C}^{3}\right)}^{2} \\
& \leq c_{1}\|u\|_{H^{1}\left(G \backslash \Gamma^{\beta} ; \mathbb{C}^{3}\right)}^{2} \\
& \leq c_{2} \int_{G \backslash \Gamma^{\beta}}|\epsilon(u)|_{\mathbb{C}^{3 \times 3}}^{2} \mathrm{~d} x
\end{aligned}
$$

for $\varphi \in P_{4} H^{\frac{1}{2}}\left(\Gamma^{\beta} ; \mathbb{C}^{3}\right)$ and suitable $c_{1}, c_{2}>0$. This proves the lower estimate.

Now we are able to give the second description of $B_{\Gamma}$.

Lemma 6.6. We have

$$
B_{\Gamma}=\left(\gamma_{N} R\right)^{*} D^{-1}\left(\gamma_{N} R\right)
$$

Proof. Note that $D^{-1}$ is bounded in $P_{4} L^{2}\left(\Gamma^{\beta} ; \mathbb{C}^{3}\right)$ because $D$ is positive definite. We make use of the characterization of $B_{\Gamma}$ given in (5.7). Let $u \in P_{4} L^{2}\left(G ; \mathbb{C}^{3}\right)$. Then

$$
-(\Delta+\operatorname{grad} \operatorname{div})\left(\gamma_{N} R\right)^{*} D^{-1}\left(\gamma_{N} R\right) u=0 \quad \text { on } \quad G \backslash \Gamma^{\beta},
$$

which follows from (6.10). Furthermore,

$$
\gamma_{N}\left(\gamma_{N} R\right)^{*}\left(-\gamma_{N}\left(\gamma_{N} R\right)^{*}\right)^{-1}\left(\gamma_{N} R\right) u=-\left(\gamma_{N} R\right) u \quad \text { on } \quad \Gamma^{\beta} .
$$

Therefore, $\left(\gamma_{N} R\right)^{*} D^{-1}\left(\gamma_{N} R\right) u$ is a solution of (5.7). Since the solution is unique, it equals $B_{\Gamma} u$.

In the following we describe the behavior of $B_{\Gamma}$ on wave functions corresponding to the spectral minimum of $A^{(4)}$. This is done with the help of the results of Subsection 6.3 .

We define $W_{\lambda}: P_{4} L^{2}\left(\Gamma^{\beta} ; \mathbb{C}^{3}\right) \rightarrow G(0)$ for almost every $\lambda \in \Theta$ by

$$
W_{\lambda} u:=J_{\lambda} \Pi_{c}\left(\gamma_{N} R\right)^{*} D^{-\frac{1}{2}} u=U_{\lambda}\left[\Pi_{0} \Pi_{c}\left(\gamma_{N} R\right)^{*} D^{-\frac{1}{2}} u\right]_{M_{\lambda}}
$$

for $u \in P_{4} L^{2}\left(\Gamma^{\beta} ; \mathbb{C}^{3}\right)$. Recall that $w_{\xi}(x)=\psi_{1}\left(\xi, x_{3}\right) \mathrm{e}^{i \xi \cdot\left(\begin{array}{l}x_{1} \\ x_{2}\end{array}\right)}$, as stated in (3.13). 
Lemma 6.7. For $u \in P_{4} L^{2}\left(\Gamma^{\beta} ; \mathbb{C}^{3}\right)$ we have

$$
W_{\lambda} u=U_{\lambda}\left[\left(2 \pi \lambda_{1}(\xi)\right)^{-1}\left\langle D^{-\frac{1}{2}} u, \gamma_{N} w_{\xi}\right\rangle_{L^{2}\left(\Gamma^{\beta} ; \mathbb{C}^{3}\right)}\right]_{\xi \in M_{\lambda}} .
$$

Proof. The proof is accomplished by showing that

$$
\left(\Pi_{0} \Pi_{c}\left(\gamma_{N} R\right)^{*} D^{-\frac{1}{2}} u\right)(\xi)=\left(2 \pi \lambda_{1}(\xi)\right)^{-1}\left\langle D^{-\frac{1}{2}} u, \gamma_{N} w_{\xi}\right\rangle_{L^{2}\left(\Gamma^{\beta} ; \mathbb{C}^{3}\right)}, \quad \xi \in \Xi,
$$

in the $L^{2}(\Xi)$-sense. Note that

$$
\left(\Pi_{0} \Pi_{c}\left(\gamma_{N} R\right)^{*} D^{-\frac{1}{2}} u\right)(\xi)=\left\langle\Phi_{\left(\begin{array}{l}
x_{1} \\
x_{2}
\end{array}\right) \rightarrow \xi}\left(\gamma_{N} R\right)^{*} D^{-\frac{1}{2}} u, \psi_{1}(\xi, \cdot)\right\rangle_{L^{2}\left(J ; \mathbb{C}^{3}\right)} .
$$

We apply an approximation argument which makes use of the following cutoff function.

Choose $\widetilde{\zeta} \in \mathrm{C}^{\infty}(\mathbb{R})$ with $\widetilde{\zeta}(t)=1$ for $t<1, \widetilde{\zeta}(t)=0$ for $t>2$, and $0 \leq \widetilde{\zeta}(t) \leq 1$ for $t \in(1,2)$ and set

$$
\zeta_{\varepsilon}(x):=\widetilde{\zeta}(\varepsilon \ln |x|) \quad \text { for } \quad x \in \mathbb{R}^{2}, \varepsilon>0 .
$$

Then $\zeta_{\varepsilon} \in \mathrm{C}_{c}^{\infty}\left(\mathbb{R}^{2}\right)$ and

$$
\int_{\mathbb{R}^{2}}\left|\nabla^{k} \zeta_{\varepsilon}\right|^{2} \mathrm{~d} x \rightarrow 0 \quad \text { as } \quad \varepsilon \rightarrow 0, \quad k=1,2
$$

We have

$$
\begin{aligned}
& \left\|\left\langle\Phi\left(1-\zeta_{\varepsilon}\right)\left(\gamma_{N} R\right)^{*} D^{-\frac{1}{2}} u, \psi_{1}\right\rangle_{L^{2}\left(J ; \mathbb{C}^{3}\right)}\right\|_{L^{2}(\Xi)}^{2} \\
& \quad \leq \int_{\mathbb{R}^{2}}\left\|\Phi\left(1-\zeta_{\varepsilon}\right)\left(\gamma_{N} R\right)^{*} D^{-\frac{1}{2}} u\right\|_{L^{2}\left(J ; \mathbb{C}^{3}\right)}^{2}\left\|\psi_{1}\right\|_{L^{2}\left(J ; \mathbb{C}^{3}\right)}^{2} \mathrm{~d} \xi \\
& \quad=\left\|\left(1-\zeta_{\varepsilon}\right)\left(\gamma_{N} R\right)^{*} D^{-\frac{1}{2}} u\right\|_{L^{2}\left(G ; \mathbb{C}^{3}\right)}^{2} .
\end{aligned}
$$

Since $\left(\gamma_{N} R\right)^{*} D^{-\frac{1}{2}} u \in L^{2}\left(G ; \mathbb{C}^{3}\right)$, the last expression tends to zero as $\varepsilon \rightarrow 0$. Therefore,

$$
\left\langle\Phi \zeta_{\varepsilon}\left(\gamma_{N} R\right)^{*} D^{-\frac{1}{2}} u, \psi_{1}\right\rangle_{L^{2}\left(J ; \mathbb{C}^{3}\right)} \rightarrow\left\langle\Phi\left(\gamma_{N} R\right)^{*} D^{-\frac{1}{2}} u, \psi_{1}\right\rangle_{L^{2}\left(J ; \mathbb{C}^{3}\right)}
$$

in $L^{2}(\Xi)$ as $\varepsilon \rightarrow 0$. On the other hand,

$$
\begin{aligned}
\left\langle\Phi \zeta_{\varepsilon}\left(\gamma_{N} R\right)^{*} D^{-\frac{1}{2}} u, \psi_{1}\right\rangle_{L^{2}\left(J ; \mathbb{C}^{3}\right)} & =(2 \pi)^{-1} \int_{G}\left\langle\left(\gamma_{N} R\right)^{*} D^{-\frac{1}{2}} u, \zeta_{\varepsilon} w_{\xi}\right\rangle_{\mathbb{C}^{3}} \mathrm{~d} x \\
& =(2 \pi)^{-1}\left\langle D^{-\frac{1}{2}} u, \gamma_{N} R\left(\zeta_{\varepsilon} w_{\xi}\right)\right\rangle_{L^{2}\left(\Gamma^{\beta} ; \mathbb{C}^{3}\right)}
\end{aligned}
$$

Therefore, it remains to show that

$$
\left\langle D^{-\frac{1}{2}} u, \gamma_{N} R\left(\zeta_{\varepsilon} w_{\xi}\right)\right\rangle_{L^{2}\left(\Gamma^{\beta} ; \mathbb{C}^{3}\right)} \rightarrow\left(\lambda_{1}(\xi)\right)^{-1}\left\langle D^{-\frac{1}{2}} u, \gamma_{N} w_{\xi}\right\rangle_{L^{2}\left(\Gamma^{\beta} ; \mathbb{C}^{3}\right)}
$$

in $L^{2}(\Xi)$ as $\varepsilon \rightarrow 0$. This is obtained by showing that

$$
\left\|R\left(\zeta_{\varepsilon} w_{\xi}\right)-\lambda_{1}(\xi)^{-1} w_{\xi}\right\|_{H^{2}\left(M ; \mathbb{C}^{3}\right)} \rightarrow 0 \quad \text { as } \quad \varepsilon \rightarrow 0
$$

uniformly in $\xi \in \Xi$ for a suitable neighborhood $M$ of $\Gamma^{\beta}$ in $G$. Note that

$$
A^{(4)}\left(w_{\xi} \zeta_{\varepsilon}\right)=\lambda_{1}(\xi) w_{\xi} \zeta_{\varepsilon}+\sum_{k=1}^{2} a_{k} \partial_{k} \zeta_{\varepsilon}+\sum_{k, l=1}^{2} b_{k l} \partial_{k} \partial_{l} \zeta_{\varepsilon}
$$


for some functions $a_{k}, b_{k l}: G \rightarrow \mathbb{C}^{3}, k, l=1,2$, which are uniformly bounded with respect to $\xi \in \Xi$. Let $\varepsilon>0$ be so small that $\zeta_{\varepsilon}=1$ on $M$. Then by (6.8) and by the boundedness of $R$ we obtain

$$
\begin{aligned}
\left\|R\left(w_{\xi} \zeta_{\varepsilon}\right)-\lambda_{1}(\xi)^{-1} w_{\xi}\right\|_{H^{2}\left(M ; \mathbb{C}^{3}\right)} & \leq\left\|\lambda_{1}(\xi)^{-1} R\left(\sum_{k} a_{k} \partial_{k} \zeta_{\varepsilon}+\sum_{k, l} b_{k l} \partial_{k} \partial_{l} \zeta_{\varepsilon}\right)\right\|_{H^{2}\left(G ; \mathbb{C}^{3}\right)} \\
& \leq C \int_{\mathbb{R}^{2}}\left(\left|\nabla \zeta_{\varepsilon}\right|^{2}+\left|\nabla^{2} \zeta_{\varepsilon}\right|^{2}\right) \mathrm{d} x
\end{aligned}
$$

for some $C>0$ independent of $\xi \in \Xi$. Since the latter expression tends to zero as $\varepsilon \rightarrow 0$, this proves (6.19).

Lemma 6.7 shows that the operator $X: P_{4} L^{2}\left(\Gamma^{\beta} ; \mathbb{C}^{3}\right) \rightarrow L^{2}\left(M_{0}, \mathrm{~d} \mu_{0}\right)$ given by

$$
X u(\xi):=W_{0} u(\xi)=\frac{1}{2 \pi \Lambda}\left\langle D^{-\frac{1}{2}} u, \gamma_{N} w_{\xi}\right\rangle_{L^{2}\left(\Gamma^{\beta} ; \mathbb{C}^{3}\right)}, \quad \xi \in M_{0},
$$

for $u \in P_{4} L^{2}\left(\Gamma^{\beta} ; \mathbb{C}^{3}\right)$ is well defined. Moreover, there exists a constant $C>0$ such that

$$
\left\|X u-W_{\lambda} u\right\|_{G(0)}^{2} \leq C \lambda^{2}\|u\|_{L^{2}\left(\Gamma^{\beta} ; \mathbb{C}^{3}\right)}^{2} \quad \text { for } \quad u \in P_{4} L^{2}\left(\Gamma^{\beta} ; \mathbb{C}^{3}\right), \lambda \in \Theta .
$$

The essential spectral properties of the Birman-Schwinger operator (6.3) can be described in terms of the operator

$$
\mathcal{L}(\tau):=F^{*} \mathcal{K}(\tau) \mathcal{K}^{*}(\tau) F \oplus \mathbb{O} \quad \text { on } \quad \Pi_{c} \mathcal{H}_{4} \oplus\left(\mathrm{I}-\Pi_{c}\right) \mathcal{H}_{4},
$$

where $\mathcal{K}(\tau)$ is given by

$$
\mathcal{K}(\tau): P_{4} L^{2}\left(\Gamma^{\beta} ; \mathbb{C}^{3}\right) \rightarrow G_{\Theta}: u \mapsto \eta_{\tau} \otimes X u .
$$

The function

$$
\eta_{\tau}(\lambda)=\left(\frac{\left(\lambda^{2}+\Lambda\right)(\Lambda-\tau)}{\lambda^{2}+\tau}\right)^{\frac{1}{2}}=\left(\frac{\tau}{\Lambda(\Lambda-\tau)}+\frac{1}{\Lambda}-\frac{1}{\lambda^{2}+\Lambda}\right)^{-\frac{1}{2}}, \quad \lambda \in \Theta,
$$

corresponds to the outer terms of the Birman-Schwinger operator (6.3). The spectrum of $\mathcal{L}(\tau)$ is basically determined by the spectrum of

$$
K=X X^{*} \text {. }
$$

Note that this is a compact selfadjoint integral operator in $L^{2}\left(M_{0}, \mathrm{~d} \mu_{0}\right)$.

\section{$\S 7$. Proof of the main estimate}

In this section we prove Theorem 4.2

7.1. Proof for circular boundaries. At the first step, we prove Theorem 4.2 for circular boundary perturbations $\Gamma^{\beta}=\left\{x \in \mathbb{R}^{2}:|x|=\beta\right\} \times J$ for $\beta>0$.

In the sequel, we use the notation

$$
\varrho(\tau):=\frac{1}{w^{-1}(\tau)}, \quad \pi(\tau):=\frac{1}{v^{-1}(\tau)},
$$

where $w(t)=t^{-2 t}$ and $v(t)=t^{-t}$ for $t>1$, while for $0<t \leq 1$ we put $w(t)=t^{-1}$ and $v(t)=t^{-1 / 2}$. Note that $\pi(\tau)=\varrho\left(\tau^{2}\right)$. Furthermore, in [15] it was shown that

$$
\lim _{\tau \rightarrow 0+} \frac{\varrho(\tau)}{\varrho(c \tau)}=1, \quad c>0
$$

In 88 below we shall prove that the operator $K$ defined in (6.25) satisfies

$$
\Delta_{\pi}(K)=1, \quad \delta_{\pi}(K)=1,
$$


with the functionals $\Delta_{\pi}$ and $\delta_{\pi}$ as in $₫ 6$. Let $\left\{\lambda_{j}(K)\right\}_{j \in \mathbb{N}}$ denote the eigenvalues of $K$, in the nonincreasing order including multiplicities. Using the minimax principle [24, 9.2.4], from (6.22) we see that the $j$ th eigenvalue of $\mathcal{L}(\tau)$ fulfills

$$
\lambda_{j}(\mathcal{L}(\tau))=\left\|\eta_{\tau}\right\|_{L^{2}(\Theta)}^{2} \lambda_{j}(K) .
$$

Note that

$$
\zeta_{\tau}:=\sqrt{\tau}\left\|\eta_{\tau}\right\|_{L^{2}(\Theta)}^{2} \rightarrow \pi \Lambda^{2} \quad \text { as } \quad \tau \rightarrow 0
$$

and

$$
n(1, \alpha \mathcal{L}(\tau))=n\left(\left(\alpha \zeta_{\tau}\right)^{-1} \sqrt{\tau}, K\right), \quad \alpha>0 .
$$

Here $n(s, T)$ denotes the number of singular values of $T$ strictly larger than $s$ (including multiplicities). Thus, using (7.2), we get

$$
\begin{aligned}
\Delta_{\varrho}(\alpha \mathcal{L}) & =\limsup _{\tau \rightarrow 0+} \varrho(\tau) n(1, \alpha \mathcal{L}(\tau)) \\
& =\limsup _{\tau \rightarrow 0+} \frac{\varrho(\tau)}{\varrho\left(\left(\alpha \zeta_{\tau}\right)^{-2} \tau\right)} \varrho\left(\left[\left(\alpha \zeta_{\tau}\right)^{-1} \sqrt{\tau}\right]^{2}\right) n\left(\left(\alpha \zeta_{\tau}\right)^{-1} \sqrt{\tau}, K\right)=\Delta_{\pi}(K)=1 .
\end{aligned}
$$

In the same way we show that $\delta_{\varrho}(\alpha \mathcal{L})=1$. From the definition of $\mathcal{L}$ in (6.22), it follows that $n(1, \alpha \mathcal{L}(\tau))=n\left(1, \sqrt{\alpha} F^{*} \mathcal{K}(\tau)\right)$. Therefore,

$$
\Delta_{\varrho}\left(\alpha F^{*} \mathcal{K}\right)=1, \quad \delta_{\varrho}\left(\alpha F^{*} \mathcal{K}\right)=1 \quad \text { for all } \quad \alpha>0 .
$$

We introduce

$$
\mathcal{Z}(\tau):=D^{-\frac{1}{2}}\left(\gamma_{N} R\right)\left(\frac{\tau}{\Lambda(\Lambda-\tau)}+\frac{1}{\Lambda}-R\right)^{-\frac{1}{2}} .
$$

Then the Birman-Schwinger operator (6.3) equals $\mathcal{Y}(\tau)=\mathcal{Z}^{*}(\tau) \mathcal{Z}(\tau)$. As in 15, Lemma 9.1], it can be checked that $\mathcal{Z}^{*}(\tau)-F^{*} \mathcal{K}(\tau)$ converges in $\mathfrak{S}_{\infty}$ to a compact operator as $\tau \rightarrow 0$. Therefore, we can apply Lemma 6.2 for this operator family to show that

$$
\left(\mathcal{Z}^{*}(\tau)-F^{*} \mathcal{K}(\tau)\right) \in \mathcal{S}_{0}^{\varrho} .
$$

By (7.4), we have $\alpha F^{*} \mathcal{K} \in \mathcal{S}^{\varrho}$ and $\Delta_{\varrho}\left(\alpha F^{*} \mathcal{K}\right)=1$ for all $\alpha>0$. Hence, Lemma 6.1 is applicable, which yields

$$
\Delta_{\varrho}(\mathcal{Y})=\Delta_{\varrho}\left(\mathcal{Z}^{*}\right)=\Delta_{\varrho}\left(F^{*} \mathcal{K}\right)=1 .
$$

Similarly, $\delta_{\varrho}(\mathcal{Y})=1$. The proof is completed by applying the Birman-Schwinger principle (6.4).

7.2. Proof for arbitrary boundaries. We use the result for circular boundaries to derive the asymptotics for general boundary perturbations.

Since the problem is invariant under translations of the first two variables, the proof of Subsection 7.1 shows that Theorem 4.2 holds true for boundary perturbations of the type $\Gamma^{\beta}(y)=\left\{x \in \mathbb{R}^{2}:|x-y|=\beta\right\} \times J$ with $\beta>0$ and $y \in \mathbb{R}^{2}$. In order to prove Theorem 4.2 for general boundaries $\Gamma=\Gamma_{0} \times J$, first we show that

$$
\frac{n_{-}\left(\Lambda-\tau, A_{\Gamma}^{(4)}\right)}{w^{-1}(\tau)} \leq \lim _{\tau \rightarrow 0+} \frac{n_{-}\left(\Lambda-\tau, A_{\Gamma_{e}}^{(4)}\right)}{w^{-1}(\tau)}=1,
$$

where $\Gamma_{e}=\Gamma^{\beta}(0)$ with $\beta>\sup \left\{|x|: x \in \Gamma_{0}\right\}$.

Consider the operator $A_{\Gamma \cup \Gamma_{e}}^{(4)}$ associated with the quadratic form

$$
a_{\Gamma \cup \Gamma_{e}}^{(4)}[u]=2 \int_{G \backslash\left(\Gamma \cup \Gamma_{e}\right)}|\epsilon(u)|_{\mathbb{C}^{3 \times 3}}^{2} \mathrm{~d} x, \quad u \in D\left[a_{\Gamma \cup \Gamma_{e}}^{(4)}\right],
$$


where $D\left[a_{\Gamma \cup \Gamma_{e}}^{(4)}\right]:=P_{4} H^{1}\left(G \backslash\left(\Gamma \cup \Gamma_{e}\right) ; \mathbb{C}^{3}\right)$. Arguing as in 23 , we can check that the essential spectrum of $A_{\Gamma \cup \Gamma_{e}}^{(4)}$ coincides with that of $A_{\Gamma}^{(4)}$. Since $D\left[a_{\Gamma}^{(4)}\right] \subset D\left[a_{\Gamma \cup \Gamma_{e}}^{(4)}\right]$ and $a_{\Gamma}^{(4)}=a_{\Gamma \cup \Gamma_{e}}^{(4)}$ on $D\left[a_{\Gamma}^{(4)}\right]$, we have $A_{\Gamma \cup \Gamma_{e}}^{(4)}<A_{\Gamma}^{(4)}$. Thus,

$$
n_{-}\left(\Lambda-\tau, A_{\Gamma}^{(4)}\right) \leq n_{-}\left(\Lambda-\tau, A_{\Gamma \cup \Gamma_{e}}^{(4)}\right), \quad \tau \in(0, \Lambda) .
$$

On the other hand, the discrete spectra of $A_{\Gamma \cup \Gamma_{e}}^{(4)}$ and $A_{\Gamma_{e}}^{(4)}$ below $\Lambda$ differ only by finitely many eigenvalues. To see this, we observe that both operators admit orthogonal expansions into operators acting on $G \backslash \overline{\mathrm{B}_{\beta}(0)}$ and $\mathrm{B}_{\beta}(0)$, respectively. Here $\mathrm{B}_{\beta}(y)=\left\{x \in \mathbb{R}^{2}\right.$ : $|x-y|<\beta\} \times J$. In both cases, the parts on $\mathrm{B}_{\beta}(0)$ have discrete spectrum accumulating at infinity only and therefore having a finite number of eigenvalues below $\Lambda$. But the parts on the unbounded domain coincide. Thus,

$$
\lim _{\tau \rightarrow 0+} \frac{n_{-}\left(\Lambda-\tau, A_{\Gamma}^{(4)}\right)}{w^{-1}(\tau)} \leq \lim _{\tau \rightarrow 0+} \frac{n_{-}\left(\Lambda-\tau, A_{\Gamma \cup \Gamma_{e}}^{(4)}\right)}{w^{-1}(\tau)}=\lim _{\tau \rightarrow 0+} \frac{n_{-}\left(\Lambda-\tau, A_{\Gamma_{e}}^{(4)}\right)}{w^{-1}(\tau)}=1 .
$$

The corresponding lower estimate can be verified much as the upper estimate by reduction of the problem to some circular boundary $\Gamma_{\beta}(y)$. Here $\beta>0$ and $y \in \mathbb{R}^{2}$ are chosen so that $\Gamma_{\beta}(y)$ lies in the bounded part of $G$ bordered by $\Gamma$.

\section{§8. Treatment of the operator $K$}

In this section, we estimate the eigenvalues of the operator $K$ given in (6.25) for the case of a circular boundary perturbation $\Gamma^{\beta}=S_{\beta} \times J$ with $S_{\beta}=\left\{x \in \mathbb{R}^{2}:|x|=\beta\right\}$. From (6.20) we obtain

$$
K=\widetilde{X} D^{-1} \widetilde{X}^{*}
$$

where

$$
\widetilde{X} u(\xi):=(2 \pi \Lambda)^{-1}\left\langle u, \gamma_{N} w_{\xi}\right\rangle_{L^{2}\left(\Gamma^{\beta} ; \mathbb{C}^{3}\right)}, \quad \xi \in M_{0},
$$

for $u \in P_{4} L^{2}\left(\Gamma^{\beta} ; \mathbb{C}^{3}\right)$. We estimate the operator $D$ with the help of simple model operators $T_{1}$ and $T_{2}$, which reflect the main spectral properties of $D$. Let

$$
\varphi_{n}(x):=\left(2 \pi^{2} \beta\right)^{-\frac{1}{2}} \mathrm{e}^{i n \cdot \tau^{-1}(x)}=\left(2 \pi^{2} \beta\right)^{-\frac{1}{2}} \mathrm{e}^{i n_{1} \mu^{-1}\left(x_{1}, x_{2}\right)} \mathrm{e}^{i n_{2}\left(2 x_{3}+\pi\right)}
$$

for $x \in \Gamma^{\beta}=S_{\beta} \times J$ and $n \in \mathbb{Z}^{2}$, where the mappings $\tau:[0,2 \pi]^{2} \rightarrow \Gamma^{\beta}$ and $\mu:[0,2 \pi] \rightarrow$ $S_{\beta}$ are given by

$$
\tau(s, t)=(\beta \cos s, \beta \sin s,(t-\pi) / 2)^{T} \quad \text { and } \quad \mu(s)=(\beta \cos s, \beta \sin s) .
$$

The functions $\varphi_{n}, n \in \mathbb{Z}^{2}$, constitute an orthonormal basis of $L^{2}\left(\Gamma^{\beta}\right)$. We define two quadratic forms $t_{1}$ and $t_{2}$ by

$$
t_{j}[u]:=\sum_{n \in \mathbb{Z}^{2}}\left(1+\left|n_{1}\right|+\delta_{j 2}\left|n_{2}\right|\right)\left|\int_{\Gamma^{\beta}} u(x) \overline{\varphi_{n}(x)} \mathrm{dS}_{\Gamma^{\beta}}(x)\right|_{\mathbb{C}^{3}}^{2}
$$

for suitable $u \in P_{4} L^{2}\left(\Gamma^{\beta} ; \mathbb{C}^{3}\right)$. It is easily seen that $t_{1}$ and $t_{2}$ are well defined on the spaces

$$
\begin{aligned}
& D_{1}:=P_{4} H^{1}\left(\Gamma^{\beta} ; \mathbb{C}^{3}\right), \\
& D_{2}:=\left\{u \in P_{4} H^{1}\left(\Gamma^{\beta} ; \mathbb{C}^{3}\right): u(\cdot, \cdot,-\pi / 2)=u(\cdot, \cdot, \pi / 2)\right\} .
\end{aligned}
$$

Denote by $D\left[t_{j}\right]$ for $j=1,2$ the completion of $D_{j}$ with respect to the norm $u \mapsto \sqrt{t_{j}[u]}$. Then $t_{j}$ admits a unique extension up to a closed positive definite form on $D\left[t_{j}\right]$, which 
is again denoted by $t_{j}$. It is associated with a positive definite selfadjoint operator $T_{j}$ in $P_{4} L^{2}\left(\Gamma_{\beta} ; \mathbb{C}^{3}\right)$ given by

$$
\left(T_{j} u\right)(x)=\sum_{n \in \mathbb{Z}^{2}}\left(1+\left|n_{1}\right|+\delta_{j 2}\left|n_{2}\right|\right) \varphi_{n}(x) \int_{\Gamma^{\beta}} u(y) \overline{\varphi_{n}(y)} \mathrm{dS}_{\Gamma^{\beta}}(y), \quad x \in \Gamma^{\beta},
$$

for suitable $u \in P_{4} L^{2}\left(\Gamma^{\beta} ; \mathbb{C}^{3}\right)$. Note that the inverse of $T_{j}$ is given by

$$
\left(T_{j}^{-1} u\right)(x)=\sum_{n \in \mathbb{Z}^{2}}\left(1+\left|n_{1}\right|+\delta_{j 2}\left|n_{2}\right|\right)^{-1} \varphi_{n}(x) \int_{\Gamma^{\beta}} u(y) \overline{\varphi_{n}(y)} \mathrm{dS}_{\Gamma^{\beta}}(y), \quad x \in \Gamma^{\beta} .
$$

Lemma 8.1. We have

$$
\widetilde{c}^{-1} T_{1} \leq D \leq \widetilde{c} T_{2}
$$

in the quadratic form sense for some $\widetilde{c}>0$.

The proof of this lemma is rather technical; we present it in the Appendix.

Set $\widetilde{K}_{j}:=\widetilde{X} T_{j}^{-1} \widetilde{X}^{*}$ for $j=1,2$ and recall that $K=\widetilde{X} D^{-1} \widetilde{X}^{*}$. The above lemma shows that

$$
\widetilde{c}^{-1} \widetilde{K}_{2} \leq K \leq \widetilde{c} \widetilde{K}_{1}
$$

This allows us to study the operators $\widetilde{K}_{j}$ instead of $K$.

8.1. Calculation of the eigenvalues. Note that each $\widetilde{K}_{j}, j=1,2$, is an integral operator in $L^{2}\left(M_{0}, \mathrm{~d} \mu_{0}\right)$ with the kernel

$$
\widetilde{k}_{j}(\eta, \xi)=(2 \pi \Lambda)^{-2}\left\langle T_{j}^{-1} \gamma_{N} w_{\xi}, \gamma_{N} w_{\eta}\right\rangle_{L^{2}\left(\Gamma^{\beta} ; \mathbb{C}^{3}\right)}, \quad \eta, \xi \in M_{0} .
$$

Since $\mathbf{n}_{\Gamma^{\beta}}(x)=\beta^{-1}\left(x_{1}, x_{2}, 0\right)$ for $x \in \Gamma^{\beta}$, from the definition of $w_{\xi}$ in (3.13) and of $\gamma_{N}$ in (5.6) we obtain

$$
\gamma_{N} w_{\xi}(x)=\left(2 p \beta^{2}\right)^{-\frac{1}{2}} \xi \cdot\left(\begin{array}{c}
x_{1} \\
x_{2}
\end{array}\right) \mathrm{e}^{i \xi \cdot\left(\begin{array}{c}
x_{1} \\
x_{2}
\end{array}\right)}\left(\begin{array}{c}
-2 \xi d_{1}\left(x_{3}\right) \\
i\left(d_{1}^{\prime}\left(x_{3}\right)+d_{2}\left(x_{3}\right)\right)
\end{array}\right), \quad x \in \Gamma^{\beta},
$$

where $p=\varkappa^{2}\left\|d_{1}\right\|_{L^{2}(J)}^{2}+\left\|d_{2}\right\|_{L^{2}(J)}^{2}$ and $d_{j}(t)=d_{j}(\varkappa, t)$ for $j=1,2$. Thus, (8.5) and passage to the cylindrical coordinates $(s, t) \in(0,2 \pi)^{2}$ for integration over $x \in \Gamma^{\beta}$ show that

$$
\widetilde{k}_{j}(\eta, \xi)=\left(64 \pi^{4} \Lambda^{2} p \beta\right)^{-1} \sum_{n \in \mathbb{Z}^{2}}\left(1+\left|n_{1}\right|+\delta_{j 2}\left|n_{2}\right|\right)^{-1} I_{n_{1}}(\xi) \overline{I_{n_{1}}(\eta)} J_{n_{2}}(\xi) \cdot \overline{J_{n_{2}}(\eta)}
$$

with

$$
I_{k}(\xi)=\int_{0}^{2 \pi} \mathrm{e}^{-i k s} i \beta\left(\xi_{1} \cos s+\xi_{2} \sin s\right) \mathrm{e}^{i \beta\left(\xi_{1} \cos s+\xi_{2} \sin s\right)} \mathrm{d} s
$$

and

With the substitutions

$$
J_{k}(\xi)=\int_{0}^{2 \pi} \mathrm{e}^{-i k t}\left(\begin{array}{c}
-2 \xi d_{1}\left(\frac{t-\pi}{2}\right) \\
i\left(d_{1}^{\prime}\left(\frac{t-\pi}{2}\right)+d_{2}\left(\frac{t-\pi}{2}\right)\right)
\end{array}\right) \mathrm{d} t
$$

$$
\eta=\varkappa\left(\begin{array}{c}
\cos v \\
\sin v
\end{array}\right), \quad \xi=\varkappa\left(\begin{array}{c}
\cos w \\
\sin w
\end{array}\right), \quad \mathrm{d} \mu_{0}=\frac{\varkappa}{|\nabla P|} \mathrm{d} v=\frac{\varkappa}{q} \mathrm{~d} v
$$

we can regard the operator $\widetilde{K}_{j}$ also as an operator in $L^{2}((0,2 \pi))$. Using the identity $\eta \cdot \xi=\varkappa^{2} \cos (w-v)$, we see that

$$
J_{k}(\xi) \cdot \overline{J_{k}(\eta)}=\sum_{m=-1}^{1} p_{|m|}(k) \mathrm{e}^{i m(w-v)}
$$


where

$$
p_{0}(k)=\left|\int_{-\pi}^{\pi} \mathrm{e}^{-i k t}\left(d_{1}^{\prime}\left(\frac{t}{2}\right)+d_{2}\left(\frac{t}{2}\right)\right) \mathrm{d} t\right|^{2}, \quad p_{1}(k)=2 \varkappa^{2}\left|\int_{-\pi}^{\pi} \mathrm{e}^{-i k t} d_{1}\left(\frac{t}{2}\right) \mathrm{d} t\right|^{2} .
$$

Furthermore, if we expand the second exponential function in 8.10 in a Taylor series and apply (8.11), we get

$$
I_{k}(\xi)=I_{k}(w)=\mathrm{e}^{-i k w} 2 \pi \sum_{l=\delta_{0 k}}^{\infty}\left(\frac{i \beta \varkappa}{2}\right)^{2 l+|k|} \frac{2 l+|k|}{l !(l+|k|) !} .
$$

Therefore, the kernel of $\widetilde{K}_{j}$ as an operator in $L^{2}((0,2 \pi))$ is given by

$$
\widetilde{k}_{j}(v, w)=\sum_{n_{1}=-\infty}^{\infty} \mu_{\left|n_{1}\right|}^{j} \frac{\mathrm{e}^{i n_{1}(w-v)}}{2 \pi},
$$

where

$$
\begin{aligned}
\mu_{k}^{j}=\frac{\varkappa}{8 \pi \Lambda^{2} p q \beta} \sum_{m=-1}^{1} & \left(\sum_{n_{2} \in \mathbb{Z}} \frac{p_{|m|}\left(n_{2}\right)}{1+|k-m|+\delta_{j 2}\left|n_{2}\right|}\right) \\
& \times\left(\sum_{l=\delta_{0, k-m}}^{\infty}\left(\frac{\beta \varkappa}{2}\right)^{2 l+|k-m|} \frac{(-1)^{l}(2 l+|k-m|)}{l !(l+|k-m|) !}\right)^{2}
\end{aligned}
$$

for $k \in \mathbb{N}_{0}$. This means that $\widetilde{K}_{j}$ has exactly the eigenfunctions $v \mapsto \mathrm{e}^{ \pm i k v}$ corresponding to the eigenvalues $\mu_{k}^{j}$ for $k \in \mathbb{N}_{0} 3$

8.2. Estimates for the eigenvalues. A detailed analysis of the expression (8.14) (to be given in the Appendix) shows that we can find $c>0$ and $N \in \mathbb{N}$ such that

$$
c^{-1}(2 k)^{2}\left(\frac{\beta \varkappa \mathrm{e}}{2 k}\right)^{2 k} \leq \mu_{k}^{2} \leq \mu_{k}^{1} \leq c(2 k)^{2}\left(\frac{\beta \varkappa \mathrm{e}}{2 k}\right)^{2 k} \quad \text { for all } \quad k>N .
$$

By (8.13) the eigenspaces of the eigenvalues $\mu_{k}^{j}$ have dimension 2 for $k \geq 1$ and dimension 1 for $k=0$. We denote by $\lambda_{n}(K), n \in \mathbb{N}$, the eigenvalues of the operator $K$ in the nonincreasing order including multiplicities. Then from (8.7) and (8.15) we see that there exists a constant $c>0$ such that

$$
c^{-1} n^{2}\left(\frac{\beta \varkappa \mathrm{e}}{n}\right)^{n} \leq \lambda_{n+1}(K) \leq \lambda_{n}(K) \leq c n^{2}\left(\frac{\beta \varkappa \mathrm{e}}{n}\right)^{n}
$$

for all sufficiently large $n \in 2 \mathbb{N}$.

8.3. Estimates for the number of eigenvalues. From (8.16), it is easily seen that

$$
\left(\frac{1}{c n}\right)^{n}<\lambda_{n}(K)<\left(\frac{c}{n}\right)^{n}, \quad n \in \mathbb{N},
$$

for some constant $c>0$. Proceeding as in [15], from (8.17) we obtain the desired asymptotic estimate (7.3) on the counting function for eigenvalues of $K$.

\footnotetext{
${ }^{3}$ We point out that similar types of eigenvalue asymptotics appeared, e.g., in [19]-[21].
} 


\section{$\S 9$. Appendix}

9.1. Proof of (8.15). By the definition (8.12), we have

$$
\sum_{n_{2}=-\infty}^{\infty} p_{m}\left(n_{2}\right)<\infty \text { for } m=0,1
$$

Setting

$$
C:=\frac{\varkappa}{8 \pi \Lambda^{2} p q \beta} \sum_{m=-1}^{1}\left(\frac{\beta \varkappa}{2}\right)^{-2 m} \sum_{n_{2}=-\infty}^{\infty} p_{|m|}\left(n_{2}\right),
$$

from (8.14) for $k \geq 2$ we get

$$
\mu_{k}^{1} \leq C\left(\frac{\beta \varkappa}{2}\right)^{2 k} \frac{1}{k}\left(\sum_{l=0}^{\infty}\left(\frac{\beta \varkappa}{2}\right)^{2 l} \frac{2 l+k+1}{l !(l+k-1) !}\right)^{2} .
$$

Estimating the right bracket and using Stirling's formula, we arrive at the inequality

$$
\mu_{k}^{1} \leq 9 C\left(\frac{\beta \varkappa}{2}\right)^{2 k} \frac{k^{3}}{(k !)^{2}}\left(\sum_{l=0}^{\infty}\left(\frac{\beta \varkappa}{2}\right)^{2 l} \frac{1}{l !}\right)^{2} \leq \frac{9 C \mathrm{e}^{(\beta \varkappa)^{2} / 2}}{8 \pi}(2 k)^{2}\left(\frac{\beta \varkappa \mathrm{e}}{2 k}\right)^{2 k} .
$$

This proves the upper estimate in (8.15). In order to check the lower, we set

$$
C:=\frac{\varkappa}{16 \pi \Lambda^{2} p q \beta}\left(\frac{\beta \varkappa}{2}\right)^{-2} \sum_{n_{2} \in \mathbb{Z}} \frac{p_{1}\left(n_{2}\right)}{1+\left|n_{2}\right|} .
$$

Then

$$
\begin{aligned}
\mu_{k}^{2} & \geq C\left(\frac{\beta \varkappa}{2}\right)^{2 k} \frac{1}{k-1}\left(\sum_{l=0}^{\infty}\left(\frac{\beta \varkappa}{2}\right)^{2 l} \frac{(-1)^{l}(2 l+k-1)}{l !(l+k-1) !}\right)^{2} \\
& \geq \frac{C}{2}\left(\frac{\beta \varkappa}{2}\right)^{2 k} \frac{k^{3}}{(k !)^{2}}\left|1-\frac{1}{k}\left(\frac{\beta \varkappa}{2}\right)^{2} \sum_{l=0}^{\infty}\left(\frac{\beta \varkappa}{2}\right)^{2 l} \frac{(2 l+k+1) k !}{(k-1)(l+1) !(l+k) !}\right|_{+}^{2},
\end{aligned}
$$

where $|x|_{+}=\frac{1}{2}(|x|+x)$ for $x \in \mathbb{R}$. Using the inequality $\frac{2 l+k+1}{(k-1)(l+1)} \leq 3$ for $k \geq 2$ and Stirling's formula, we obtain

$$
\mu_{k}^{2} \geq \frac{C}{16 \pi \mathrm{e}^{\frac{1}{6 n}}}\left|1-\frac{3}{k}\left(\frac{\beta \varkappa}{2}\right)^{2} \mathrm{e}^{(\beta \varkappa)^{2} / 4}\right|_{+}^{2}(2 k)^{2}\left(\frac{\beta \varkappa \mathrm{e}}{2 k}\right)^{2 k} .
$$

This proves the lower bound in (8.15) for sufficiently large $k \in \mathbb{N}$.

9.2. Proof of Lemma 8.1. As stated in Lemma 6.5, we have

$$
c^{-1}\|u\|_{H^{\frac{1}{2}}\left(\Gamma^{\beta} ; \mathbb{C}^{3}\right)}^{2} \leq\langle D u, u\rangle_{L^{2}\left(\Gamma^{\beta} ; \mathbb{C}^{3}\right)} \leq c\|u\|_{H^{\frac{1}{2}}\left(\Gamma^{\beta} ; \mathbb{C}^{3}\right)}^{2}, \quad u \in P_{4} H^{\frac{1}{2}}\left(\Gamma^{\beta} ; \mathbb{C}^{3}\right),
$$

for some $c>0$. Therefore, it suffices to prove that

$$
t_{1}[u] \leq c_{1}\|u\|_{H^{\frac{1}{2}}\left(\Gamma^{\beta} ; \mathbb{C}^{3}\right)}^{2}, \quad u \in D_{1},
$$

and

$$
t_{2}[u] \geq c_{2}\|u\|_{H^{\frac{1}{2}}\left(\Gamma^{\beta} ; \mathbb{C}^{3}\right)}^{2}, \quad u \in D_{2},
$$

for some constants $c_{1}, c_{2}>0$. We start with (9.2). Note that among several equivalent norms we may choose

$$
\|u\|_{H^{\frac{1}{2}}\left(\Gamma^{\beta} ; \mathbb{C}^{3}\right)}=\left(\|u\|_{L^{2}\left(\Gamma^{\beta} ; \mathbb{C}^{3}\right)}^{2}+|u|_{H^{\frac{1}{2}}\left(\Gamma^{\beta} ; \mathbb{C}^{3}\right)}^{2}\right)^{\frac{1}{2}},
$$


where

$$
|u|_{H^{\frac{1}{2}}\left(\Gamma^{\beta} ; \mathbb{C}^{3}\right)}^{2}=\int_{\Gamma^{\beta}} \int_{\Gamma^{\beta}} \frac{|u(x)-u(y)|^{2}}{|x-y|^{3}} \mathrm{dS}_{\Gamma^{\beta}}(x) \mathrm{dS}_{\Gamma^{\beta}}(y)
$$

denotes the Sobolev-Slobodetskiur seminorm of order 1/2; see [31. Recall that $\Gamma_{\beta}=S_{\beta} \times J$ with $S_{\beta}=\left\{x \in \mathbb{R}^{2}:|x|=\beta\right\}$. We define a new seminorm $|\cdot|_{w}$ on $H^{\frac{1}{2}}\left(\Gamma^{\beta} ; \mathbb{C}^{3}\right)$ by

$$
|u|_{w}^{2}:=\int_{J} \int_{S_{\beta}} \int_{S_{\beta}} \frac{\left|u\left(x^{\prime}, t\right)-u\left(y^{\prime}, t\right)\right|^{2}}{\left|x^{\prime}-y^{\prime}\right|^{2}} \mathrm{dS}_{S_{\beta}}\left(x^{\prime}\right) \mathrm{dS}_{S_{\beta}}\left(y^{\prime}\right) \mathrm{d} t .
$$

Roughly speaking, $|\cdot|_{w}$ measures smoothness of order $1 / 2$ only along $S_{\beta}$, whereas $|\cdot|_{H^{\frac{1}{2}}\left(\Gamma^{\beta} ; \mathbb{C}^{3}\right)}$ measures smoothness also along the third coordinate. Thus, it is reasonable to expect that

$$
\|u\|_{L^{2}\left(\Gamma^{\beta} ; \mathbb{C}^{3}\right)}^{2}+|u|_{w}^{2} \leq c\|u\|_{H^{\frac{1}{2}}\left(\Gamma^{\beta} ; \mathbb{C}^{3}\right)}^{2}, \quad u \in H^{\frac{1}{2}}\left(\Gamma^{\beta} ; \mathbb{C}^{3}\right),
$$

for some $c>0$. This is true indeed. We give an outline of the proof after we have finished the present proof.

Recall the definition of $\varphi_{n}$ given in (8.3). Applying the expansion

$$
u=\sum_{n \in \mathbb{Z}^{2}} u_{n} \varphi_{n}, \quad u_{n}=\int_{\Gamma^{\beta}} u(x) \overline{\varphi_{n}(x)} \mathrm{dS}_{\Gamma^{\beta}}(x)
$$

for $u \in P_{4} H^{\frac{1}{2}}\left(\Gamma_{\beta} ; \mathbb{C}^{3}\right)$, we see that

$$
\begin{aligned}
|u|_{w}^{2} & =\int_{J} \int_{S_{\beta}} \int_{S_{\beta}} \frac{\left|\sum_{n \in \mathbb{Z}^{2}} u_{n}\left(\varphi_{n}\left(x^{\prime}, t\right)-\varphi_{n}\left(y^{\prime}, t\right)\right)\right|^{2}}{\left|x^{\prime}-y^{\prime}\right|^{2}} \mathrm{dS}_{S_{\beta}}\left(x^{\prime}\right) \mathrm{dS}_{S_{\beta}}\left(y^{\prime}\right) \mathrm{d} t \\
& =\frac{1}{2 \pi \beta} \sum_{n_{2} \in \mathbb{Z}} \int_{S_{\beta}} \int_{S_{\beta}} \frac{\left|\sum_{n_{1} \in \mathbb{Z}} u_{n}\left(\mathrm{e}^{i n_{1} \mu^{-1}\left(x^{\prime}\right)}-\mathrm{e}^{i n_{1} \mu^{-1}\left(y^{\prime}\right)}\right)\right|^{2}}{\left|x^{\prime}-y^{\prime}\right|^{2}} \mathrm{dS}_{S_{\beta}}\left(x^{\prime}\right) \mathrm{dS}_{S_{\beta}}\left(y^{\prime}\right) .
\end{aligned}
$$

In order to prove (9.3), we note that the substitutions $x=\tau(s)$ and $y=\tau(t)$ with $s, t \in(0,2 \pi)^{2}$ yield

$$
\begin{aligned}
|u|_{H^{\frac{1}{2}}\left(\Gamma^{\beta} ; \mathbb{C}^{3}\right)}^{2} & =\int_{\Gamma^{\beta}} \int_{\Gamma^{\beta}} \frac{\left|\sum_{n \in \mathbb{Z}^{2}} u_{n}\left(\varphi_{n}(x)-\varphi_{n}(y)\right)\right|^{2}}{\left(\left|x_{1}-y_{1}\right|^{2}+\left|x_{2}-y_{2}\right|^{2}+\left|x_{3}-y_{3}\right|^{2}\right)^{\frac{3}{2}}} \mathrm{dS}_{\Gamma_{\beta}}(x) \mathrm{dS}_{\Gamma_{\beta}}(y) \\
& =\frac{\beta}{8 \pi^{2}} \int_{(0,2 \pi)^{2}} \int_{(0,2 \pi)^{2}} \frac{\left|\sum_{n \in \mathbb{Z}^{2}} u_{n}\left(\mathrm{e}^{i n \cdot s}-\mathrm{e}^{i n \cdot t}\right)\right|^{2}}{\left(4 \beta^{2} \sin ^{2}\left(\frac{s_{1}-t_{1}}{2}\right)+4^{-1}\left|s_{2}-t_{2}\right|^{2}\right)^{\frac{3}{2}}} \mathrm{~d} s \mathrm{~d} t .
\end{aligned}
$$

Using the change of variables $s=t+h$ and denoting $I=\left(-t_{1}, 2 \pi-t_{1}\right) \times\left(-t_{2}, 2 \pi-t_{2}\right)$, we arrive at

$$
\begin{aligned}
|u|_{H^{\frac{1}{2}}\left(\Gamma^{\beta} ; \mathbb{C}^{3}\right)}^{2} & \leq \frac{\beta}{8 \pi^{2}} \int_{(0,2 \pi)^{2}} \int_{I} \frac{\left|\sum_{n \in \mathbb{Z}^{2}} u_{n}\left(\mathrm{e}^{i n \cdot h}-1\right) \mathrm{e}^{i n \cdot t}\right|^{2}}{\left(4 \beta^{2} \sin ^{2} \frac{h_{1}}{2}+4^{-1}\left|h_{2}\right|^{2}\right)^{\frac{3}{2}}} \mathrm{~d} h \mathrm{~d} t \\
& \leq \frac{\beta}{2} \sum_{n \in \mathbb{Z}^{2}}\left|u_{n}\right|^{2} \int_{(-\pi, \pi)^{2}} \frac{\left|\mathrm{e}^{i n \cdot h}-1\right|^{2}}{\left(4 \beta^{2} \sin ^{2} \frac{h_{1}}{2}+4^{-1}\left|h_{2}\right|^{2}\right)^{\frac{3}{2}}} \mathrm{~d} h .
\end{aligned}
$$

Note that

$$
2 \beta^{-1}\left(4 \beta^{2} \sin ^{2} \frac{h_{1}}{2}+4^{-1}\left|h_{2}\right|^{2}\right)>c^{-1}|h|^{2}, \quad h \in(-\pi, \pi)^{2}
$$


for some $c>0$. Therefore, the substitution $h=(r \cos \phi, r \sin \phi)$ gives

$$
\begin{aligned}
|u|_{H^{\frac{1}{2}}\left(\Gamma^{\beta} ; \mathbb{C}^{3}\right)}^{2} & \leq c \sum_{n \in \mathbb{Z}^{2}}\left|u_{n}\right|^{2} \int_{|h|<\sqrt{2} \pi} \frac{\left|\mathrm{e}^{i n \cdot h}-1\right|^{2}}{|h|^{3}} \mathrm{~d} h \\
& =c \sum_{n \in \mathbb{Z}^{2}}\left|u_{n}\right|^{2} \int_{0}^{\sqrt{2} \pi} \int_{0}^{2 \pi} \frac{\left|\mathrm{e}^{i|n| r \cos \phi}-1\right|^{2}}{r^{2}} \mathrm{~d} \phi \mathrm{d} r \\
& \leq c \sum_{n \in \mathbb{Z}^{2}}|n|\left|u_{n}\right|^{2} \int_{0}^{\infty} \int_{0}^{2 \pi} \frac{\left|\mathrm{e}^{i t \cos \phi}-1\right|^{2}}{t^{2}} \mathrm{~d} \phi \mathrm{d} t .
\end{aligned}
$$

Since the integral on the right-hand side is finite and $|n| \leq\left|n_{1}\right|+\left|n_{2}\right|$, this proves (9.3).

9.3. Outline of the proof of (9.7). First, we consider the case of the entire space $\mathbb{R}^{n}$ for $n \geq 2$. Let $s \in(0,1)$. In [22, Lemma 3.15], it was proved that

$$
|u|_{H^{s}\left(\mathbb{R}^{n}\right)}^{2}=a_{s} \int_{\mathbb{R}^{n}}|\xi|^{2 s}|\Phi u(\xi)|^{2} \mathrm{~d} \xi
$$

for some constant $a_{s}>0$, where $\Phi$ denotes the unitary Fourier transformation in $\mathbb{R}^{n}$. Similarly,

$$
|u|_{w, s}^{2}=a_{s}^{\prime} \int_{\mathbb{R}^{n}}\left|\xi^{\prime}\right|^{2 s}|\Phi u(\xi)|^{2} \mathrm{~d} \xi
$$

for some $a_{s}^{\prime}>0$, where $\xi^{\prime}=\left(\xi_{1}, \ldots, \xi_{n-1}\right)$, and $|u|_{w, s}^{2}$ is given by (cf. (9.6) )

$$
|u|_{w, s}^{2}=\int_{\mathbb{R}} \int_{\mathbb{R}^{n-1}} \int_{\mathbb{R}^{n-1}} \frac{\left|u\left(x^{\prime}, t\right)-u\left(y^{\prime}, t\right)\right|^{2}}{\left|x^{\prime}-y^{\prime}\right|^{n-1+2 s}} \mathrm{~d} x^{\prime} \mathrm{d} y^{\prime} \mathrm{d} t .
$$

Therefore,

$$
|u|_{w, s}^{2} \leq a_{s}^{\prime} a_{s}^{-1}|u|_{H^{s}\left(\mathbb{R}^{n}\right)}^{2}, \quad u \in H^{s}\left(\mathbb{R}^{d}\right) .
$$

The second step is to carry over this result to domains $\Omega=\Omega_{0} \times I$, where $\Omega_{0} \subset \mathbb{R}^{n-1}$ is a Lipschitz domain and $I \subset \mathbb{R}$ is an interval. This is done by using a continuous extension operator $E: H^{s}(\Omega) \rightarrow H^{s}\left(\mathbb{R}^{n}\right)$. The existence of such an object was proved in [22]. Note that our definition of $\|\cdot\|_{H^{s}}$ corresponds to $\|\cdot\|_{W^{s}}$ in [22]. Put

$$
|u|_{w, s, \Omega}^{2}=\int_{I} \int_{\Omega_{0}} \int_{\Omega_{0}} \frac{\left|u\left(x^{\prime}, t\right)-u\left(y^{\prime}, t\right)\right|^{2}}{\left|x^{\prime}-y^{\prime}\right|^{n-1+2 s}} \mathrm{~d} x^{\prime} \mathrm{d} y^{\prime} \mathrm{d} t
$$

Then

$$
\|u\|_{L^{2}(\Omega)}^{2}+|u|_{w, s, \Omega}^{2} \leq\|E u\|_{L^{2}\left(\mathbb{R}^{n}\right)}^{2}+|E u|_{w, s}^{2} \leq c_{1}\|E u\|_{H^{s}\left(\mathbb{R}^{n}\right)}^{2} \leq c_{2}\|u\|_{H^{s}(\Omega)}^{2}
$$

for some $c_{1}, c_{2}>0$ and $u \in H^{s}(\Omega)$. Recalling the standard definition of the Sobolev spaces on a boundary via the Sobolev spaces on the preimages of its parametrization mappings [31, 2.5], we immediately obtain an analog of (9.8) for the standard Sobolev norm on a boundary. Finally, (9.7) is obtained by using the equivalence of the standard Sobolev norm and the norm (9.4), which carries over to the corresponding "weaker" norms.

\section{REFERENCES}

[1] P. Exner and P. Šeba, Bound states in curved quantum waveguides, J. Math. Phys. 30 (1989), 2574-2580. MR1019002 (90i:81194)

[2] J. Goldstone and R. L. Jaffe, Bound states in twisting tubes, Phys. Rev. B 45 (1992), 14100-14107.

[3] P. Duclos and P. Exner, Curvature-induced bound states in quantum waveguides in two and three dimensions, Rev. Math. Phys. 7 (1995), 73-102. MR.1310767 (95m:81239)

[4] W. Bulla, F. Gesztesy, W. Renger, and B. Simon, Weakly coupled bound states in quantum waveguides, Proc. Amer. Math. Soc. 125 (1997), no. 5, 1487-1495. MR1371117 (97g:81009) 
[5] T. Ekholm and H. Kovařík, Stability of the magnetic Schrödinger operator in a waveguide, Comm. Partial Differential Equations 30 (2005), 539-565. MR2153507(2007i:35193)

[6] D. V. Evans and C. M. Linton, Acoustic resonances in ducts, J. Sound Vib. 173 (1994), 85-94.

[7] D. V. Evans, M. Levitin, and D. Vassiliev, Existence theorems for trapped modes, J. Fluid Mech. 261 (1994), 21-31. MR1265871 (94m:76113)

[8] E. B. Davies and L. Parnovski, Trapped modes in acoustic waveguides, Quart. J. Mech. Appl. Math. 51 (1998), 477-492. MR1639044 (99g:76132)

[9] A. Aslanyan, L. Parnovski, and D. Vassiliev, Complex resonances in acoustic waveguides, Quart. J. Mech. Appl. Math. 53 (2000), 429-447. MR1777188(2001d:76110)

[10] N. S. A. Khallaf, L. Parnovski, and D. Vassiliev, Trapped modes in a waveguide with a long obstacle, J. Fluid Mech. 403 (2000), 251-261. MR.1742168(2000i:76117)

[11] I. Roitberg, D. Vassiliev, and T. Weidl, Edge resonance in an elastic semi-strip, Quart. J. Mech. Appl. Math. 51 (1998), no. 1, 1-13. MR1610688 (98m:73041)

[12] D. Gridin, R. V. Craster, and A. T. I. Adamou, Trapped modes in curved elastic plates, Proc. R. Soc. Lond. A 461 (2005), 1181-1197. MR2144620 (2006a:74056)

[13] V. Zernov, A. V. Pichugin, and J. Kaplunov, Eigenvalue of a semi-infinite elastic strip, Proc. R. Soc. Lond. A 462 (2006), 1255-1270. MR2216875 (2006k:74039)

[14] C. Förster and T.Weidl, Trapped modes for an elastic strip with perturbation of the material properties, Quart. J. Mech. Appl. Math. 59 (2006), 399-418. MR2250814 (2007j:74053)

[15] C. Förster, Trapped modes for an elastic plate with a perturbation of Young's modulus, Comm. Partial Differential Equations 33 (2008), no. 7-9, 1339-1367. MR2450161 (2009h:74007)

[16] B. Simon, The bound state of weakly coupled Schrödinger operators in one and two dimensions, Ann. Physics 97 (1976), 279-288. MR0404846 (53:8646)

[17] A. Laptev, O. Safronov, and T. Weidl, Bound states asymptotics for elliptic operators with strongly degenerated symbols, Nonlinear Problems in Mathematical Physics and Related Topics. I. In Honor of Academician O. A. Ladyzhenskaya, Mezhdunar. Mat. Ser., vol. 1, Tamara Rozhkovskaya, Novosibirsk, 2002, pp. 221-232; English transl., Int. Math. Ser. (N.Y.), vol. 1, Kluwer/Plenum, New York, 2002, pp. 233-246. MR1970614 (2004h:35168)

[18] G. D. Raikov and S. Warzel, Quasi-classical versus non-classical spectral asymptotics for magnetic Schrödinger operators with decreasing electric potentials, Rev. Math. Phys. 14 (2002), 1051-1072. MR:1939760(2003m:81069)

[19] M. Melgaard and G. Rozenblum, Eigenvalue asymptotics for weakly perturbed Dirac and Schrödinger operators with constant magnetic fields of full rank, Comm. Partial Differential Equations 28 (2003), no. 3-4, 697-736. MR.1978311 (2004e:81038)

[20] N. Filonov and A. Pushnitski, Spectral asymptotics of Pauli operators and orthogonal polynomials in complex domains, Comm. Math. Phys. 264 (2006), 759-772. MR2217290(2007h:81064)

[21] A. Pushnitski and G. Rozenblum, Eigenvalue clusters of the Landau Hamiltonian in the exterior of a compact domain, Doc. Math. 12 (2007), 569-586. MR2377242 (2008m:35255)

[22] W. McLean, Strongly elliptic systems and boundary integral equations, Cambridge Univ. Press, Cambridge, 2000. MR1742312 (2001a:35051)

[23] M. Sh. Birman, Perturbations of the continuous spectrum of a singular elliptic operator by varying the boundary and the boundary conditions, Vestnik Leningrad. Univ. Ser. Mat. Mekh. Astronom. 1962, vyp. 1, 22-55. (Russian) MR0138874 (25:2314)

[24] M. Sh. Birman and M. Z. Solomyak, Spectral theory of selfadjoint operators in Hilbert space, Leningrad. Univ., Leningrad, 1980, Chapters 10, 11; English transl., Math. Appl. (Soviet Ser.), Reidel, Dordrecht, 1987, Chapters 10, 11. MR0609148 (82k:47001); MR1192782 (93g:47001)

[25] O. Safronov, Discrete spectrum in a gap of the continuous spectrum for variable sign perturbations with large coupling constant, Algebra i Analiz 8 (1996), no. 2, 162-194; English transl., St. Petersburg Math. J. 8 (1997), no. 2, 307-332. MR1392031 (97e:47027)

[26] I. Ts. Gokhberg and M. G. Krein, Introduction to the theory of linear non-selfadjoint operators in Hilbert space, Nauka, Moscow, 1965; English transl., Transl. Math. Monogr., vol. 18, Amer. Math. Soc., Providence, RI, 1969. MR0220070 (36:3137) MR0246142 (39:7447)

[27] T. Vaĭdl (Weidl), General operator ideals of weak type, Algebra i Analiz 4 (1992), no. 3, 117-144; English transl., St. Petersburg Math. J. 4 (1993), no. 3, 503-525. MR1190773 (94a:47034)

[28] M. Sh. Birman, On the spectrum of singular boundary-value problems, Mat. Sb. 55 (1961), no. 2, 125-174; English transl., 11 Papers on Analysis, Amer. Math. Soc. Transl. (2), vol. 53, Amer. Math. Soc., Providence, RI, 1966, pp. 23-60. MR0142896 (26:463) MR0194283 (33:2496)

[29] J. Schwinger, On the bound states for a given potential, Proc. Nat. Acad. Sci. USA 47 (1961), 122-129. MR0129798 (23:B2833) 
[30] L. Nirenberg, Remarks on strongly elliptic partial differential equations, Comm. Pure Appl. Math. 8 (1955), 649-675. MR0075415 (17:742d)

[31] O. Steinbach, Numerische Näherungsverfahren für elliptische Randwertprobleme, Teubner Verlag, 2003; English transl., Numerical approximation methods for elliptic boundary value problems, Springer, New York, 2008. MR2361676(2008i:65271)

Institute for Analysis, Dynamics, and Modeling, Department of Mathematics and Physics, University of Stuttgart, Pfaffenwaldring 57, 70569 Stuttgart, Germany

E-mail address: foerster@mathematik.uni-stuttgart.de

Institute for Analysis, Dynamics, and Modeling, Department of Mathematics and Physics, University of Stuttgart, Pfaffenwaldring 57, 70569 Stuttgart, Germany

E-mail address: weidl@mathematik.uni-stuttgart.de

Received 1/OCT/2010

Translated by V. SLOUSHCH 